\title{
UK Renal Registry 18th Annual Report (December 2015): Appendix F Additional Data Tables for 2014 new and existing patients
}

\section{F:1 Patients starting renal replacement therapy in 2014}

Table F1.1. Number of patients on dialysis at 90 days (incident cohort $1 / 10 / 2013$ to $30 / 09 / 2014$ )

\begin{tabular}{lrrrrrr}
\hline & \multicolumn{2}{c}{ Aged $<65$} & & \multicolumn{2}{c}{ Aged $\geqslant 65$} \\
\cline { 2 - 3 } \cline { 6 - 6 } & HD & PD & & HD & PD \\
& $N$ & $N$ & & $N$ & $N$ \\
\hline England & 1,846 & 705 & & 2,204 & 516 \\
N Ireland & 37 & 10 & & 69 & 8 \\
Scotland & 196 & 47 & & 194 & 36 \\
Wales & 91 & 38 & & 164 & 27 \\
UK & $\mathbf{2 , 1 7 0}$ & $\mathbf{8 0 0}$ & & $\mathbf{2 , 6 3 1}$ & $\mathbf{5 8 7}$ \\
\hline
\end{tabular}

Table F1.3. First treatment modality (2014 incident cohort)

\begin{tabular}{llll}
\hline Centre & $\% \mathrm{HD}$ & $\% \mathrm{PD}$ & $\%$ transplant \\
\hline
\end{tabular}

\section{England}

B Heart

$\mathrm{B}$ QEH

Basldn

Bradfd

Brightn

Bristol

Camb

Carlis

Carsh

Chelms

Colchr

Covnt

Derby

Donc

Dorset

Dudley

Exeter

Glouc

Hull

Ipswi

Kent

$\begin{array}{rr}82 & 14 \\ 71 & 22 \\ 78 & 20 \\ 82 & 5 \\ 72 & 22 \\ 76 & 14 \\ 69 & 9 \\ 55 & 42 \\ 79 & 17 \\ 62 & 38 \\ 100 & \\ 63 & 28 \\ 51 & 44 \\ 83 & 17 \\ 68 & 26 \\ 54 & 46 \\ 71 & 24 \\ 53 & 47 \\ 68 & 23 \\ 73 & 27 \\ 72 & 22\end{array}$

\begin{tabular}{lccc}
\hline Centre & \% HD & \% PD & \% transplant \\
\hline Prestn & 76 & 19 & 5 \\
Redng & 64 & 32 & 5 \\
Salford & 69 & 30 & 1 \\
Sheff & 75 & 16 & 9 \\
Shrew & 66 & 32 & 2 \\
Stevng & 85 & 10 & 5 \\
Sthend & 60 & 40 & \\
Stoke & 72 & 27 & 1 \\
Sund & 79 & 13 & 8 \\
Truro & 82 & 13 & 5 \\
Wirral & 73 & 14 & 13 \\
Wolve & 62 & 34 & 4 \\
York & 63 & 20 & 17 \\
N Ireland & & & \\
Antrim & 94 & 6 & \\
Belfast & 62 & 8 & 30 \\
Newry & 84 & 16 & \\
Ulster & 80 & 20 & \\
West NI & 77 & 23 & \\
Scotland & & & \\
Abrdn & 83 & 17 & \\
Airdrie & 94 & 6 & \\
& & &
\end{tabular}

Table F1.2. Number of patients per treatment modality at 90 days (incident cohort 1/10/2013 to 30/09/2014)

\begin{tabular}{lrrrr}
\hline & HD & PD & Transplant & Died \\
\hline England & 4,050 & 1,221 & 614 & 291 \\
N Ireland & 106 & 18 & 21 & 6 \\
Scotland & 390 & 83 & 47 & 21 \\
Wales & 255 & 65 & 23 & 18 \\
UK & $\mathbf{4 , 8 0 1}$ & $\mathbf{1 , 3 8 7}$ & $\mathbf{7 0 5}$ & $\mathbf{3 3 6}$ \\
\hline
\end{tabular}

UK Renal Registry, Southmead Hospital, Southmead Road, Bristol, BS10 5NB, UK

Email: renalregistry@renalregistry.nhs.uk

\section{KARGER}

Fax +4161306 1234

E-Mail karger@karger.com www.karger.com/nef
C 2016 The UK Renal Registry Published by S. Karger AG, Basel $1660-8151 / 16 / 1325-0313 \$ 39.50 / 0$

Karger Open access

This article is licensed under the Creative Commons AttributionNonCommercial-NoDerivatives 4.0 International License (CC BYNC-ND) (http://www.karger.com/Services/OpenAccessLicense). Usage and distribution for commercial purposes as well as any distribution of modified material requires written permission. 
Table F1.3. Continued

\begin{tabular}{|c|c|c|c|c|c|c|c|}
\hline Centre & $\% \mathrm{HD}$ & $\% \mathrm{PD}$ & $\%$ transplant & Centre & $\% \mathrm{HD}$ & $\% \mathrm{PD}$ & $\%$ transplant \\
\hline L Guys & 72 & 9 & 19 & Dundee & 82 & 18 & \\
\hline L Rfree & 60 & 30 & 10 & Glasgw & 76 & 10 & 14 \\
\hline L St.G & 76 & 16 & 8 & Inverns & 64 & 36 & \\
\hline L West & 84 & 6 & 10 & Klmarnk & 75 & 25 & \\
\hline Liv Ain & 75 & 22 & 3 & Bangor & 73 & 27 & \\
\hline Liv Roy & 55 & 23 & 21 & Cardff & 74 & 17 & 9 \\
\hline M RI & 56 & 24 & 20 & Clwyd & 72 & 21 & 7 \\
\hline Middlbr & 78 & 9 & 14 & Swanse & 80 & 18 & 2 \\
\hline Newc & 73 & 22 & 6 & Wrexm & 76 & 21 & 2 \\
\hline Norwch & 86 & 14 & & England & 71 & 21 & 8 \\
\hline
\end{tabular}

Table F1.4. First treatment modality, patient numbers (2014 incident cohort)

\begin{tabular}{lrrr}
\hline & HD & PD & Transplant \\
\hline England & 4,486 & 1,305 & 533 \\
N Ireland & 131 & 22 & 19 \\
Scotland & 424 & 81 & 37 \\
Wales & 278 & 69 & 20 \\
UK & $\mathbf{5 , 3 1 9}$ & $\mathbf{1 , 4 7 7}$ & $\mathbf{6 0 9}$ \\
\hline
\end{tabular}

Table F1.5. Gender breakdown by treatment modality at 90 days (2014 incident cohort)

\begin{tabular}{|c|c|c|c|c|c|c|}
\hline \multirow[b]{2}{*}{ Centre } & \multicolumn{3}{|c|}{$\mathrm{HD}$} & \multicolumn{3}{|c|}{ PD } \\
\hline & $\%$ male & $\%$ female & M:F Ratio & $\%$ male & $\%$ female & M:F Ratio \\
\hline \multicolumn{7}{|l|}{ England } \\
\hline B Heart & 60 & 40 & 1.5 & 67 & 33 & 2.0 \\
\hline $\mathrm{B} \mathrm{QEH}$ & 61 & 39 & 1.6 & 59 & 42 & 1.4 \\
\hline Basldn & 62 & 39 & 1.6 & 83 & 17 & 5.0 \\
\hline Bradfd & 66 & 35 & 1.9 & 75 & 25 & 3.0 \\
\hline Brightn & 66 & 34 & 1.9 & 77 & 23 & 3.3 \\
\hline Bristol & 61 & 39 & 1.6 & 65 & 35 & 1.9 \\
\hline Camb & 65 & 35 & 1.9 & 75 & 25 & 3.0 \\
\hline Carlis & 55 & 45 & 1.2 & 63 & 38 & 1.7 \\
\hline Carsh & 64 & 36 & 1.8 & 54 & 46 & 1.2 \\
\hline Chelms & 64 & 36 & 1.8 & 77 & 23 & 3.3 \\
\hline Colchr & 58 & 42 & 1.4 & & & \\
\hline Covnt & 68 & 32 & 2.2 & 67 & 33 & 2.0 \\
\hline Derby & 62 & 38 & 1.6 & 79 & 21 & 3.8 \\
\hline Donc & 72 & 28 & 2.5 & 69 & 31 & 2.2 \\
\hline Dorset & 67 & 33 & 2.1 & 71 & 29 & 2.4 \\
\hline Dudley & 68 & 32 & 2.1 & 52 & 48 & 1.1 \\
\hline Exeter & 69 & 32 & 2.2 & 47 & 53 & 0.9 \\
\hline Glouc & 65 & 35 & 1.9 & 65 & 35 & 1.9 \\
\hline Hull & 70 & 30 & 2.4 & 72 & 28 & 2.6 \\
\hline Ipswi & 86 & 14 & 6.4 & 75 & 25 & 3.0 \\
\hline Kent & 62 & 38 & 1.6 & 68 & 32 & 2.1 \\
\hline
\end{tabular}


Table F1.5. Continued

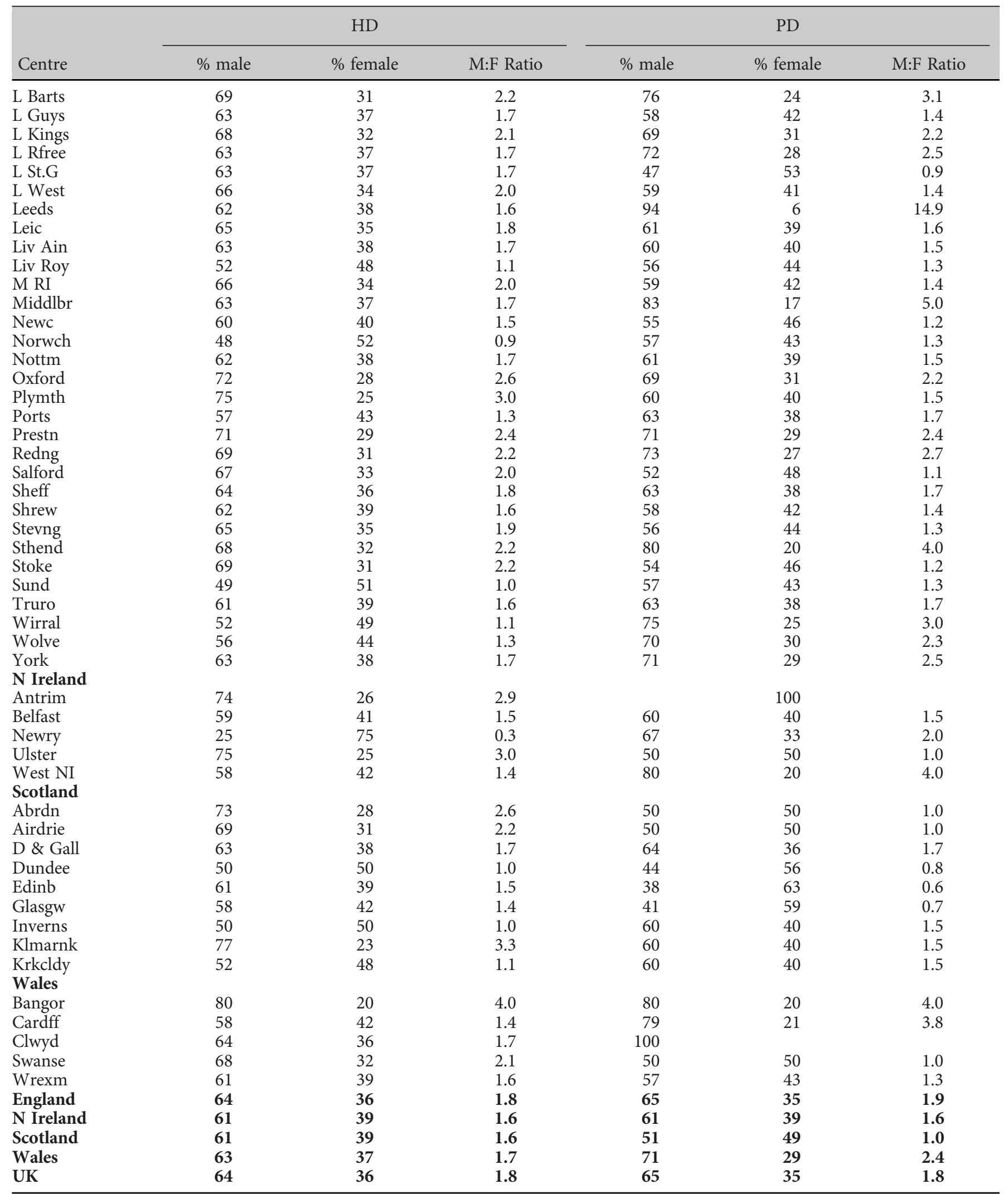


F:2 Prevalent patients on 31/12/2014

Table F2.1. Treatment modalities for 2014 prevalent patients aged under and 65 and over

\begin{tabular}{|c|c|c|c|c|c|c|c|c|}
\hline \multirow[b]{2}{*}{ Centre } & \multicolumn{4}{|c|}{ Patients aged $<65$} & \multicolumn{4}{|c|}{ Patients aged $\geqslant 65$} \\
\hline & $\% \mathrm{HD}$ & $\% \mathrm{PD}$ & $\%$ transplant & HD:PD & $\% \mathrm{HD}$ & $\% \mathrm{PD}$ & $\%$ transplant & HD:PD \\
\hline B Heart & 54 & 5 & 41 & 10.6 & 79 & 6 & 15 & 14.0 \\
\hline B QEH & 35 & 6 & 59 & 5.8 & 64 & 8 & 28 & 8.1 \\
\hline Basldn & 51 & 11 & 38 & 4.8 & 75 & 9 & 16 & 8.1 \\
\hline Bristol & 21 & 4 & 75 & 5.3 & 60 & 5 & 34 & 10.9 \\
\hline Camb & 14 & 1 & 85 & 11.0 & 57 & 5 & 38 & 12.2 \\
\hline Carlis & 19 & 8 & 73 & 2.3 & 48 & 16 & 36 & 2.9 \\
\hline Carsh & 36 & 8 & 56 & 4.6 & 69 & 10 & 21 & 7.0 \\
\hline Chelms & 39 & 9 & 53 & 4.5 & 66 & 12 & 22 & 5.4 \\
\hline Colchr & 100 & 0 & 0 & 0.0 & 100 & 0 & 0 & 0.0 \\
\hline Dudley & 47 & 20 & 33 & 2.4 & 69 & 15 & 15 & 4.5 \\
\hline Exeter & 25 & 8 & 67 & 3.2 & 66 & 12 & 22 & 5.3 \\
\hline Glouc & 28 & 11 & 61 & 2.6 & 70 & 9 & 21 & 7.5 \\
\hline Hull & 28 & 9 & 63 & 3.2 & 63 & 11 & 26 & 5.7 \\
\hline Ipswi & 27 & 5 & 69 & 5.9 & 46 & 14 & 40 & 3.2 \\
\hline Kent & 24 & 5 & 71 & 4.9 & 62 & 9 & 29 & 7.2 \\
\hline L Barts & 35 & 8 & 57 & 4.2 & 65 & 16 & 19 & 4.1 \\
\hline L Guys & 26 & 1 & 73 & 23.0 & 56 & 3 & 41 & 20.4 \\
\hline L Kings & 45 & 8 & 48 & 5.9 & 66 & 11 & 23 & 6.0 \\
\hline L Rfree & 23 & 5 & 72 & 4.3 & 59 & 11 & 31 & 5.6 \\
\hline L St.G & 30 & 4 & 66 & 7.6 & 54 & 10 & 36 & 5.3 \\
\hline L West & 32 & 1 & 66 & 24.3 & 65 & 3 & 32 & 20.4 \\
\hline Nottm & 20 & 7 & 74 & 3.0 & 60 & 10 & 30 & 5.9 \\
\hline Oxford & 18 & 3 & 78 & 5.5 & 49 & 8 & 43 & 5.8 \\
\hline Plymth & 15 & 6 & 79 & 2.5 & 45 & 10 & 45 & 4.7 \\
\hline Ports & 29 & 4 & 67 & 7.4 & 56 & 7 & 37 & 8.2 \\
\hline Prestn & 37 & 4 & 59 & 9.3 & 66 & 7 & 28 & 10.1 \\
\hline Redng & 28 & 8 & 64 & 3.4 & 54 & 12 & 35 & 4.6 \\
\hline Salford & 35 & 8 & 57 & 4.2 & 57 & 11 & 32 & 5.3 \\
\hline Sheff & 30 & 4 & 67 & 8.5 & 66 & 6 & 27 & 10.3 \\
\hline Shrew & 44 & 10 & 46 & 4.2 & 70 & 8 & 22 & 9.1 \\
\hline Stevng & 48 & 2 & 49 & 20.8 & 80 & 5 & 16 & 16.5 \\
\hline Sthend & 36 & 6 & 57 & 5.6 & 62 & 11 & 27 & 5.9 \\
\hline Stoke & 30 & 8 & 63 & 3.8 & 65 & 15 & 20 & 4.3 \\
\hline Sund & 37 & 4 & 60 & 9.7 & 65 & 4 & 30 & 14.9 \\
\hline Truro & 25 & 4 & 70 & 5.7 & 55 & 7 & 38 & 8.2 \\
\hline Wirral & 78 & 11 & 10 & 7.0 & 88 & 8 & 0 & 11.4 \\
\hline Wolve & 43 & 11 & 46 & 3.7 & 73 & 17 & 10 & 4.2 \\
\hline York & 21 & 6 & 74 & 3.4 & 51 & 7 & 42 & 7.4 \\
\hline
\end{tabular}


Table F2.1. Continued

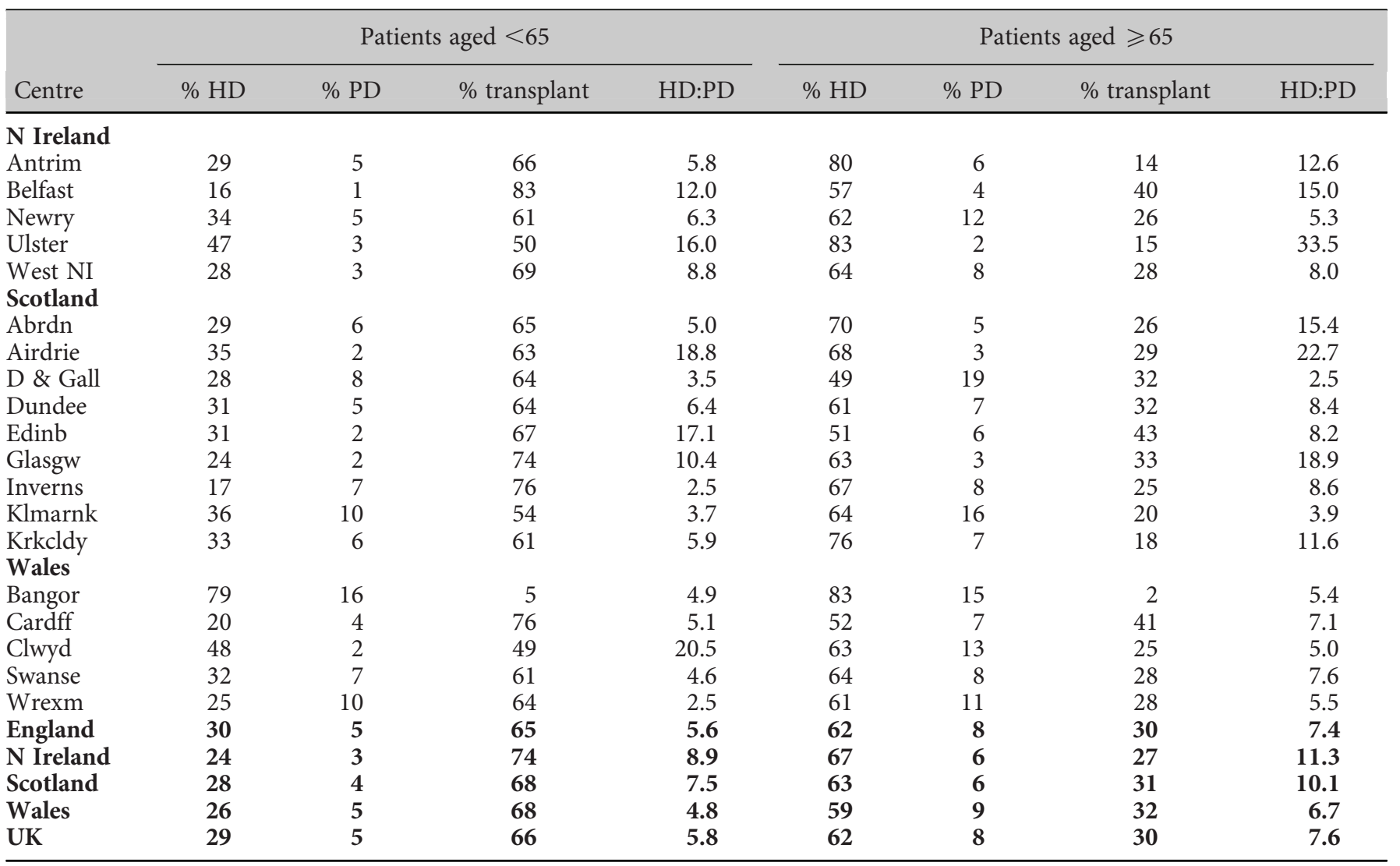

Table F2.2. Number of 2014 prevalent patients under and 65 and over per treatment modality

\begin{tabular}{|c|c|c|c|c|c|c|}
\hline & HD & $\mathrm{PD}$ & Transplant & $\mathrm{HD}$ & $\mathrm{PD}$ & Transplant \\
\hline $\mathrm{N}$ Ireland & 239 & 27 & 750 & 395 & 35 & 162 \\
\hline Scotland & 889 & 119 & 2,143 & 963 & 95 & 467 \\
\hline Wales & 447 & 93 & 1,169 & 668 & 100 & 368 \\
\hline
\end{tabular}


Table F2.3. Dialysis modalities for 2014 prevalent patients aged under 65

\begin{tabular}{|c|c|c|c|c|c|c|}
\hline Centre & $\begin{array}{c}\% \\
\text { home HD }\end{array}$ & $\begin{array}{c}\% \\
\text { hospital HD }\end{array}$ & $\begin{array}{c}\% \\
\text { satellite HD }\end{array}$ & $\begin{array}{c}\% \\
\text { CAPD }\end{array}$ & $\begin{array}{l}\% \text { unknown } \\
\text { type of PD }\end{array}$ & $\begin{array}{c}\% \\
\mathrm{APD}\end{array}$ \\
\hline \multicolumn{7}{|l|}{ England } \\
\hline $\mathrm{B}$ QEH & 7 & 13 & 65 & 5 & 0 & 10 \\
\hline Basldn & 0 & 78 & 4 & 6 & 0 & 11 \\
\hline Bradfd & 3 & 79 & 8 & 3 & 0 & 7 \\
\hline Camb & 12 & 46 & 34 & 0 & 8 & 0 \\
\hline Carlis & 0 & 49 & 21 & 7 & 0 & 23 \\
\hline Carsh & 4 & 28 & 49 & 4 & 0 & 13 \\
\hline Chelms & 0 & 82 & 0 & 9 & 3 & 6 \\
\hline Colchr & 0 & 100 & 0 & 0 & 0 & 0 \\
\hline Covnt & 6 & 70 & 0 & 25 & 0 & 0 \\
\hline Exeter & 2 & 13 & 61 & 14 & 0 & 10 \\
\hline Glouc & 2 & 60 & 10 & 4 & 0 & 24 \\
\hline Hull & 5 & 39 & 33 & 13 & 0 & 10 \\
\hline Ipswi & 1 & 72 & 12 & 4 & 0 & 10 \\
\hline Kent & 9 & 28 & 46 & 16 & 0 & 1 \\
\hline L Barts & 2 & 40 & 39 & 2 & 0 & 17 \\
\hline L Guys & 12 & 10 & 73 & 2 & 0 & 2 \\
\hline L Kings & 3 & 20 & 62 & 3 & 0 & 11 \\
\hline L Rfree & 3 & 4 & 75 & 5 & 0 & 14 \\
\hline L St.G & 2 & 44 & 42 & 2 & 1 & 8 \\
\hline L West & 2 & 22 & 71 & 2 & 0 & 2 \\
\hline Leeds & 6 & 21 & 59 & 1 & 0 & 13 \\
\hline Oxford & 6 & 31 & 47 & 3 & 0 & 12 \\
\hline Plymth & 5 & 65 & 2 & 9 & 0 & 20 \\
\hline Ports & 13 & 22 & 54 & 12 & 0 & 0 \\
\hline Prestn & 8 & 21 & 61 & 1 & 0 & 8 \\
\hline Redng & 4 & 33 & 41 & 14 & 1 & 8 \\
\hline Salford & 5 & 32 & 44 & 8 & 0 & 12 \\
\hline Sheff & 13 & 34 & 42 & 11 & 0 & 0 \\
\hline Shrew & 11 & 47 & 23 & 15 & 0 & 4 \\
\hline Stevng & 9 & 25 & 61 & 5 & 0 & 0 \\
\hline Sthend & 0 & 85 & 0 & 15 & 0 & 0 \\
\hline Stoke & 15 & 42 & 23 & 2 & 2 & 17 \\
\hline Sund & 1 & 62 & 28 & 3 & 0 & 6 \\
\hline Truro & 10 & 42 & 33 & 3 & 0 & 12 \\
\hline Wirral & 7 & 40 & 40 & 0 & 0 & 12 \\
\hline Wolve & 7 & 38 & 33 & 12 & 2 & 7 \\
\hline York & 14 & 35 & 29 & 16 & 0 & 6 \\
\hline \multicolumn{7}{|c|}{ N Ireland } \\
\hline Antrim & 2 & 83 & 0 & 0 & 0 & 15 \\
\hline Belfast & 13 & 79 & 0 & 1 & 0 & 7 \\
\hline
\end{tabular}


Table F2.3. Continued

\begin{tabular}{|c|c|c|c|c|c|c|}
\hline Centre & $\begin{array}{c}\% \\
\text { home HD }\end{array}$ & $\begin{array}{c}\% \\
\text { hospital HD }\end{array}$ & $\begin{array}{c}\% \\
\text { satellite HD }\end{array}$ & $\begin{array}{c}\% \\
\text { CAPD }\end{array}$ & $\begin{array}{l}\% \text { unknown } \\
\text { type of PD }\end{array}$ & $\begin{array}{c}\% \\
\mathrm{APD}\end{array}$ \\
\hline Newry & 2 & 84 & 0 & 0 & 0 & 14 \\
\hline Ulster & 9 & 85 & 0 & 0 & 0 & 6 \\
\hline West NI & 4 & 86 & 0 & 0 & 0 & 10 \\
\hline \multicolumn{7}{|l|}{ Scotland ${ }^{*}$} \\
\hline Abrdn & 3 & 80 & 0 & 6 & 0 & 10 \\
\hline Airdrie & 0 & 95 & 0 & 3 & 0 & 2 \\
\hline D \& Gall & 7 & 70 & 0 & 15 & 0 & 7 \\
\hline Dundee & 4 & 82 & 0 & 8 & 0 & 6 \\
\hline Edinb & 3 & 92 & 0 & 2 & 0 & 4 \\
\hline Glasgw & 8 & 84 & 0 & 2 & 0 & 7 \\
\hline Inverns & 5 & 67 & 0 & 18 & 0 & 10 \\
\hline Klmarnk & 4 & 74 & 0 & 1 & 0 & 20 \\
\hline Krkcldy & 0 & 85 & 0 & 0 & 0 & 15 \\
\hline \multicolumn{7}{|l|}{ Wales } \\
\hline Bangor & 27 & 41 & 15 & 7 & 0 & 10 \\
\hline Cardff & 12 & 15 & 58 & 10 & 0 & 6 \\
\hline Clwyd & 5 & 91 & 0 & 2 & 0 & 2 \\
\hline Swanse & 21 & 37 & 25 & 13 & 0 & 5 \\
\hline Wrexm & 2 & 59 & 10 & 0 & 0 & 29 \\
\hline England & 7 & 34 & 44 & 6 & 0 & 9 \\
\hline N Ireland & 7 & 83 & 0 & 0 & $\mathbf{0}$ & 10 \\
\hline Scotland* & 4 & 84 & 0 & 4 & 0 & 8 \\
\hline Wales & 14 & 34 & 36 & 9 & 0 & 8 \\
\hline UK & 7 & 39 & 39 & 6 & 0 & 9 \\
\hline
\end{tabular}

*All haemodialysis patients in centres in Scotland are shown as receiving treatment at home or in centre as no data is available regarding satellite dialysis

Table F2.4. Dialysis modalities for 2014 prevalent patients aged 65 and over

\begin{tabular}{ccccccc}
\hline & $\%$ & $\%$ & $\%$ & $\%$ & $\%$ unknown & $\%$ \\
Centre & home HD & hospital HD & satellite HD & CAPD & type of PD & APD \\
\hline
\end{tabular}

\section{England}

B Heart

B QEH

Basldn

Bradfd

Brightn

Bristol

Camb

Carlis

Carsh

Chelms

Colchr

Covnt

Derby

Donc

Dorset

Dudley

Exeter

Glouc

Hull

Ipswi

Kent

L Barts

L Guys

L Kings

83

10

86

67

38

14

42

51

18

83

100

83

69

44

18

57

10

67

38

61

25

38

15

15

9
77
3
25
45
75
47
24
68
0
0
0
0
45
66
24
74
20
47
12
62
42
78
71

71

5
4
6
2
10
5
0
17
3
11
0
16
14
1
4
14
6
3
7
11
9
5
2
8

1

5

3

4

0

8

9

4

0

0

6

10

10

4

9

9

7

12

3

15

3 
Table F2.4. Continued

\begin{tabular}{|c|c|c|c|c|c|c|}
\hline Centre & $\begin{array}{c}\% \\
\text { home HD }\end{array}$ & $\begin{array}{c}\% \\
\text { hospital HD }\end{array}$ & $\begin{array}{c}\% \\
\text { satellite HD }\end{array}$ & $\begin{array}{c}\% \\
\text { CAPD }\end{array}$ & $\begin{array}{l}\% \text { unknown } \\
\text { type of PD }\end{array}$ & $\begin{array}{c}\% \\
\mathrm{APD}\end{array}$ \\
\hline L Rfree & 1 & 2 & 81 & 7 & 0 & 8 \\
\hline L West & 0 & 22 & 73 & 3 & 0 & 2 \\
\hline Leeds & 0 & 13 & 79 & 1 & 0 & 7 \\
\hline Leic & 4 & 15 & 71 & 4 & 0 & 7 \\
\hline M RI & 2 & 23 & 62 & 4 & 0 & 9 \\
\hline Middlbr & 2 & 20 & 75 & 4 & 0 & 0 \\
\hline Newc & 1 & 83 & 0 & 3 & 0 & 14 \\
\hline Norwch & 6 & 48 & 38 & 6 & 0 & 1 \\
\hline Nottm & 2 & 40 & 43 & 7 & 0 & 7 \\
\hline Oxford & 1 & 33 & 51 & 3 & 0 & 12 \\
\hline Salford & 1 & 25 & 58 & 5 & 0 & 10 \\
\hline Sheff & 1 & 40 & 50 & 9 & 0 & 0 \\
\hline Shrew & 3 & 52 & 35 & 7 & 0 & 2 \\
\hline Stevng & 2 & 24 & 68 & 6 & 0 & 0 \\
\hline Sthend & 1 & 84 & 0 & 14 & 0 & 0 \\
\hline Stoke & 3 & 50 & 28 & 3 & 7 & 9 \\
\hline Sund & 0 & 60 & 33 & 3 & 0 & 4 \\
\hline Truro & 3 & 43 & 44 & 7 & 0 & 4 \\
\hline Wirral & 1 & 42 & 49 & 1 & 0 & 7 \\
\hline Wolve & 2 & 37 & 42 & 10 & 4 & 5 \\
\hline York & 0 & 33 & 55 & 11 & 0 & 1 \\
\hline \multicolumn{7}{|l|}{ N Ireland } \\
\hline D \& Gall & 0 & 72 & 0 & 21 & 0 & 8 \\
\hline Dundee & 0 & 89 & 0 & 6 & 0 & 4 \\
\hline Edinb & 1 & 88 & 0 & 3 & 0 & 8 \\
\hline Glasgw & 1 & 93 & 0 & 1 & 0 & 4 \\
\hline Inverns & 2 & 88 & 0 & 8 & 0 & 2 \\
\hline Klmarnk & 8 & 72 & 0 & 2 & 0 & 18 \\
\hline Krkcldy & 0 & 92 & 0 & 1 & 0 & 7 \\
\hline \multicolumn{7}{|l|}{ Wales } \\
\hline Bangor & 3 & 43 & 38 & 9 & 0 & 7 \\
\hline Cardff & 2 & 11 & 74 & 9 & 0 & 3 \\
\hline Clwyd & 5 & 78 & 0 & 8 & 0 & 8 \\
\hline Swanse & 4 & 48 & 37 & 10 & 0 & 2 \\
\hline Wrexm & 0 & 70 & 14 & 0 & 0 & 15 \\
\hline England & 2 & 33 & 54 & 6 & 0 & 6 \\
\hline N Ireland & 1 & 91 & $\mathbf{0}$ & $\mathbf{0}$ & 0 & 7 \\
\hline Scotland* & 2 & 90 & 0 & 3 & 0 & 6 \\
\hline Wales & 3 & 37 & 47 & 8 & 0 & 5 \\
\hline UK & 2 & 39 & 48 & 6 & $\mathbf{0}$ & 6 \\
\hline
\end{tabular}

* All haemodialysis patients in centres in Scotland are shown as receiving treatment at home or in centre as no data is available regarding satellite dialysis 
Table F2.5. Prevalent patient 2014, age ranges by centre (\%)

\begin{tabular}{|c|c|c|c|c|c|c|c|c|}
\hline Centre & $18-24$ & $25-34$ & $35-44$ & $45-54$ & $55-64$ & $65-74$ & $75-84$ & $85+$ \\
\hline \multicolumn{9}{|l|}{ England } \\
\hline B Heart & 1 & 5 & 12 & 17 & 20 & 21 & 20 & 3 \\
\hline $\mathrm{B} \mathrm{QEH}$ & 3 & 7 & 12 & 22 & 24 & 18 & 12 & 2 \\
\hline Basldn & 3 & 5 & 10 & 18 & 18 & 24 & 18 & 5 \\
\hline Bradfd & 3 & 10 & 15 & 22 & 21 & 17 & 10 & 1 \\
\hline Brightn & 1 & 5 & 11 & 20 & 21 & 22 & 16 & 3 \\
\hline Bristol & 3 & 7 & 11 & 19 & 21 & 22 & 14 & 3 \\
\hline Camb & 2 & 7 & 14 & 20 & 20 & 20 & 13 & 3 \\
\hline Carlis & 2 & 8 & 8 & 22 & 23 & 20 & 15 & 2 \\
\hline Carsh & 1 & 5 & 10 & 21 & 19 & 23 & 16 & 5 \\
\hline Chelms & 2 & 5 & 7 & 15 & 25 & 23 & 17 & 6 \\
\hline Colchr & 2 & 3 & 3 & 7 & 15 & 30 & 33 & 8 \\
\hline Covnt & 2 & 7 & 13 & 22 & 21 & 20 & 14 & 3 \\
\hline Derby & 1 & 8 & 9 & 20 & 20 & 26 & 14 & 2 \\
\hline Donc & 2 & 4 & 8 & 16 & 22 & 22 & 21 & 4 \\
\hline Dorset & 1 & 6 & 8 & 17 & 18 & 26 & 20 & 4 \\
\hline Dudley & 1 & 5 & 6 & 19 & 22 & 23 & 19 & 5 \\
\hline Exeter & 2 & 6 & 8 & 17 & 20 & 24 & 16 & 6 \\
\hline Glouc & 1 & 4 & 10 & 17 & 17 & 26 & 17 & 7 \\
\hline Hull & 2 & 7 & 11 & 21 & 22 & 20 & 14 & 2 \\
\hline Ipswi & 1 & 5 & 10 & 21 & 23 & 24 & 12 & 4 \\
\hline Kent & 2 & 5 & 10 & 20 & 20 & 24 & 15 & 3 \\
\hline L Barts & 2 & 8 & 15 & 23 & 24 & 17 & 10 & 1 \\
\hline L Guys & 4 & 9 & 15 & 23 & 22 & 16 & 8 & 2 \\
\hline L Kings & 1 & 5 & 12 & 22 & 23 & 19 & 15 & 3 \\
\hline L Rfree & 2 & 9 & 13 & 21 & 21 & 19 & 13 & 3 \\
\hline L St.G & 1 & 5 & 13 & 19 & 25 & 20 & 13 & 3 \\
\hline L West & 1 & 6 & 12 & 20 & 26 & 21 & 12 & 2 \\
\hline Leeds & 3 & 8 & 13 & 23 & 21 & 19 & 11 & 1 \\
\hline Leic & 2 & 6 & 12 & 22 & 20 & 22 & 13 & 3 \\
\hline Liv Ain & 1 & 3 & 9 & 17 & 17 & 21 & 24 & 8 \\
\hline Liv Roy & 3 & 8 & 14 & 24 & 24 & 18 & 8 & 1 \\
\hline M RI & 4 & 8 & 14 & 25 & 22 & 19 & 9 & 1 \\
\hline Middlbr & 2 & 7 & 11 & 22 & 21 & 22 & 12 & 3 \\
\hline Newc & 3 & 7 & 12 & 23 & 22 & 20 & 11 & 1 \\
\hline Norwch & 2 & 5 & 8 & 20 & 21 & 23 & 16 & 5 \\
\hline Nottm & 4 & 7 & 12 & 21 & 20 & 20 & 13 & 3 \\
\hline Oxford & 2 & 7 & 14 & 24 & 21 & 18 & 11 & 3 \\
\hline Plymth & 2 & 6 & 11 & 17 & 24 & 24 & 12 & 3 \\
\hline Ports & 1 & 7 & 11 & 22 & 23 & 21 & 14 & 2 \\
\hline Prestn & 1 & 6 & 11 & 21 & 22 & 24 & 13 & 1 \\
\hline Redng & 1 & 4 & 12 & 18 & 23 & 23 & 15 & 3 \\
\hline Salford & 2 & 6 & 14 & 23 & 23 & 20 & 13 & 1 \\
\hline Sheff & 2 & 7 & 11 & 21 & 23 & 19 & 13 & 3 \\
\hline Shrew & 1 & 5 & 10 & 20 & 19 & 24 & 17 & 3 \\
\hline Stevng & 2 & 5 & 9 & 21 & 18 & 21 & 21 & 3 \\
\hline Sthend & 2 & 5 & 10 & 16 & 20 & 25 & 18 & 5 \\
\hline Stoke & 1 & 8 & 13 & 18 & 21 & 20 & 15 & 4 \\
\hline Sund & 1 & 5 & 14 & 22 & 23 & 23 & 11 & 1 \\
\hline Truro & 2 & 5 & 11 & 17 & 19 & 23 & 19 & 4 \\
\hline Wirral & 2 & 4 & 8 & 17 & 16 & 26 & 22 & 5 \\
\hline Wolve & 1 & 6 & 11 & 19 & 23 & 19 & 18 & 3 \\
\hline York & 4 & 9 & 12 & 18 & 22 & 19 & 12 & 3 \\
\hline \multicolumn{9}{|l|}{ N Ireland } \\
\hline Antrim & 1 & 5 & 10 & 17 & 19 & 22 & 21 & 4 \\
\hline Belfast & 4 & 8 & 14 & 25 & 20 & 15 & 11 & 2 \\
\hline Newry & 2 & 6 & 13 & 19 & 24 & 21 & 14 & 1 \\
\hline Ulster & 1 & 3 & 13 & 11 & 17 & 21 & 23 & 9 \\
\hline West NI & 1 & 8 & 16 & 19 & 15 & 24 & 16 & 1 \\
\hline
\end{tabular}


Table F2.5. Continued

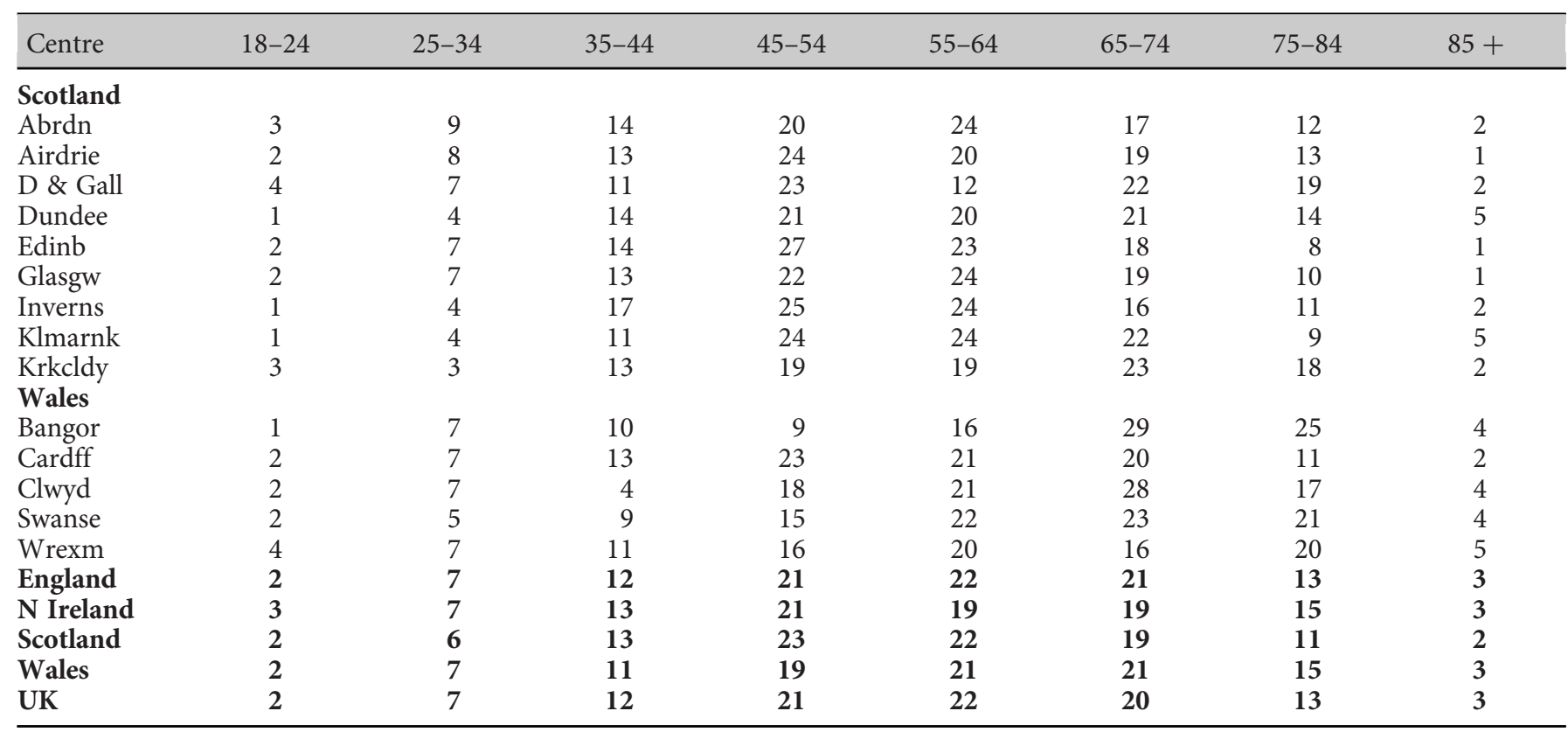

Table F2.6. Dialysis modalities for 2014 prevalent patients without diabetes (all ages)

\begin{tabular}{|c|c|c|c|c|c|c|}
\hline \multicolumn{7}{|l|}{ England } \\
\hline B QEH & 5 & 11 & 69 & 5 & 0 & 9 \\
\hline Basldn & 0 & 85 & 3 & 5 & 0 & 6 \\
\hline Bradfd & 3 & 70 & 16 & 3 & 0 & 8 \\
\hline Camb & 6 & 41 & 45 & 0 & 8 & 0 \\
\hline Carlis & 0 & 46 & 22 & 16 & 0 & 16 \\
\hline Carsh & 4 & 17 & 66 & 3 & 0 & 10 \\
\hline Chelms & 1 & 85 & 0 & 11 & 0 & 3 \\
\hline Covnt & 3 & 78 & 0 & 19 & 0 & 0 \\
\hline Glouc & 2 & 68 & 15 & 4 & 0 & 11 \\
\hline Hull & 3 & 34 & 44 & 11 & 0 & 8 \\
\hline Ipswi & 3 & 67 & 12 & 8 & 0 & 10 \\
\hline Kent & 4 & 26 & 56 & 11 & 0 & 2 \\
\hline L Barts & 2 & 40 & 40 & 3 & 0 & 15 \\
\hline L Guys & 11 & 11 & 75 & 1 & 0 & 2 \\
\hline L Kings & 2 & 17 & 66 & 6 & 0 & 9 \\
\hline L Rfree & 3 & 3 & 78 & 6 & 0 & 10 \\
\hline L St.G & 2 & 36 & 47 & 4 & 1 & 10 \\
\hline L West & 2 & 21 & 73 & 3 & 0 & 2 \\
\hline Leeds & 4 & 17 & 68 & 1 & 0 & 11 \\
\hline
\end{tabular}


Table F2.6. Continued

\begin{tabular}{|c|c|c|c|c|c|c|}
\hline Centre & $\begin{array}{c}\% \\
\text { home HD }\end{array}$ & $\begin{array}{c}\% \\
\text { hospital HD }\end{array}$ & $\begin{array}{c}\% \\
\text { satellite HD }\end{array}$ & $\begin{array}{c}\% \\
\text { CAPD }\end{array}$ & $\begin{array}{l}\% \text { unknown } \\
\text { type of PD }\end{array}$ & $\begin{array}{c}\% \\
\mathrm{APD}\end{array}$ \\
\hline Liv Ain & 5 & 7 & 71 & 4 & 0 & 14 \\
\hline M RI & 11 & 24 & 53 & 4 & 0 & 8 \\
\hline Middlbr & 5 & 27 & 64 & 4 & 0 & 0 \\
\hline Newc & 8 & 78 & 0 & 1 & 0 & 14 \\
\hline Oxford & 4 & 31 & 49 & 3 & 0 & 12 \\
\hline Plymth & 4 & 69 & 3 & 8 & 0 & 16 \\
\hline Ports & 7 & 18 & 63 & 12 & 0 & 0 \\
\hline Prestn & 7 & 19 & 64 & 1 & 0 & 8 \\
\hline Redng & 2 & 38 & 43 & 11 & 0 & 6 \\
\hline Sthend & 0 & 86 & 0 & 14 & 0 & 0 \\
\hline Stoke & 9 & 44 & 27 & 3 & 5 & 13 \\
\hline Sund & 1 & 61 & 31 & 3 & 0 & 5 \\
\hline Truro & 4 & 39 & 44 & 6 & 0 & 6 \\
\hline Wirral & 4 & 41 & 46 & 1 & 0 & 8 \\
\hline Wolve & 5 & 36 & 39 & 11 & 2 & 7 \\
\hline York & 7 & 36 & 43 & 12 & 0 & 2 \\
\hline \multicolumn{7}{|l|}{ N Ireland } \\
\hline Antrim & 1 & 90 & 0 & 1 & 0 & 8 \\
\hline Belfast & 8 & 86 & 0 & 1 & 0 & 5 \\
\hline Newry & 3 & 82 & 0 & 0 & 0 & 16 \\
\hline Ulster & 5 & 91 & 0 & 0 & 0 & 4 \\
\hline Inverns & 4 & 79 & 0 & 12 & 0 & 4 \\
\hline Klmarnk & 8 & 71 & 0 & 1 & 0 & 20 \\
\hline Krkcldy & 0 & 90 & 0 & 1 & 0 & 10 \\
\hline \multicolumn{7}{|l|}{ Wales } \\
\hline Bangor & 17 & 40 & 24 & 8 & 0 & 11 \\
\hline Cardff & 6 & 14 & 66 & 10 & 0 & 4 \\
\hline Clwyd & 5 & 83 & 0 & 6 & 0 & 6 \\
\hline Swanse & 11 & 43 & 31 & 11 & 0 & 3 \\
\hline Wrexm & 1 & 62 & 13 & 0 & 0 & 24 \\
\hline England & 5 & 32 & 49 & 6 & 0 & 7 \\
\hline N Ireland & 4 & 86 & 0 & 1 & 0 & 8 \\
\hline Scotland ${ }^{*}$ & 3 & 87 & 0 & 3 & $\mathbf{0}$ & 7 \\
\hline Wales & 8 & 35 & 42 & 9 & 0 & 7 \\
\hline UK & 5 & 38 & 44 & 6 & 0 & 7 \\
\hline
\end{tabular}

Excluded one centre with $\geqslant 40 \%$ primary renal diagnosis aetiology uncertain (Colchester)

Patients with diabetes as their primary renal disease and patients with a missing primary renal diagnosis code are excluded from this table * All haemodialysis patients in centres in Scotland are shown as receiving treatment at home or in centre as no data is available regarding satellite dialysis 
Table F2.7. Number of 2014 prevalent patients without diabetes by treatment modality

\begin{tabular}{lrrr}
\hline & HD & PD & Transplant \\
\hline England & 14,849 & 2,276 & 22,624 \\
N Ireland & 474 & 48 & 836 \\
Scotland & 1,434 & 159 & 2,355 \\
Wales & 852 & 156 & 1,338 \\
UK & $\mathbf{1 7 , 6 0 9}$ & $\mathbf{2 , 6 3 9}$ & $\mathbf{2 7 , 1 5 3}$ \\
\hline
\end{tabular}

Excluded one centre with $\geqslant 40 \%$ primary renal diagnosis aetiology uncertain (Colchester)

Patients with diabetes as their primary renal disease and patients with a missing primary renal diagnosis code are excluded from this table

Table F2.8. Dialysis modalities for 2014 prevalent patients without diabetes aged under 65

\begin{tabular}{|c|c|c|c|c|c|c|}
\hline Centre & $\begin{array}{c}\% \\
\text { home HD }\end{array}$ & $\begin{array}{c}\% \\
\text { hospital HD }\end{array}$ & $\begin{array}{c}\% \\
\text { satellite HD }\end{array}$ & $\begin{array}{c}\% \\
\text { CAPD }\end{array}$ & $\begin{array}{l}\% \text { unknown } \\
\text { type of PD }\end{array}$ & $\begin{array}{c}\% \\
\mathrm{APD}\end{array}$ \\
\hline \multicolumn{7}{|l|}{ England } \\
\hline B QEH & 7 & 13 & 63 & 6 & 0 & 11 \\
\hline Basldn & 0 & 79 & 5 & 5 & 0 & 11 \\
\hline Bradfd & 5 & 77 & 7 & 3 & 0 & 8 \\
\hline Camb & 14 & 45 & 33 & 0 & 9 & 0 \\
\hline Carlis & 0 & 44 & 22 & 9 & 0 & 25 \\
\hline Carsh & 6 & 28 & 51 & 3 & 0 & 11 \\
\hline Chelms & 0 & 86 & 0 & 11 & 0 & 2 \\
\hline Covnt & 7 & 70 & 0 & 23 & 0 & 0 \\
\hline Exeter & 3 & 13 & 59 & 14 & 0 & 11 \\
\hline Glouc & 3 & 67 & 6 & 5 & 0 & 19 \\
\hline Hull & 6 & 34 & 36 & 15 & 0 & 8 \\
\hline Ipswi & 2 & 75 & 11 & 4 & 0 & 9 \\
\hline Kent & 11 & 27 & 45 & 16 & 0 & 1 \\
\hline L Barts & 3 & 42 & 37 & 2 & 0 & 16 \\
\hline L Guys & 17 & 9 & 71 & 1 & 0 & 2 \\
\hline L Kings & 4 & 19 & 62 & 4 & 0 & 11 \\
\hline L Rfree & 3 & 4 & 74 & 4 & 0 & 14 \\
\hline L St.G & 3 & 39 & 44 & 2 & 2 & 10 \\
\hline L West & 3 & 22 & 71 & 3 & 0 & 2 \\
\hline Leeds & 7 & 20 & 58 & 1 & 0 & 14 \\
\hline Nottm & 17 & 36 & 21 & 8 & 0 & 17 \\
\hline Oxford & 8 & 31 & 46 & 3 & 0 & 11 \\
\hline
\end{tabular}


Table F2.8. Continued

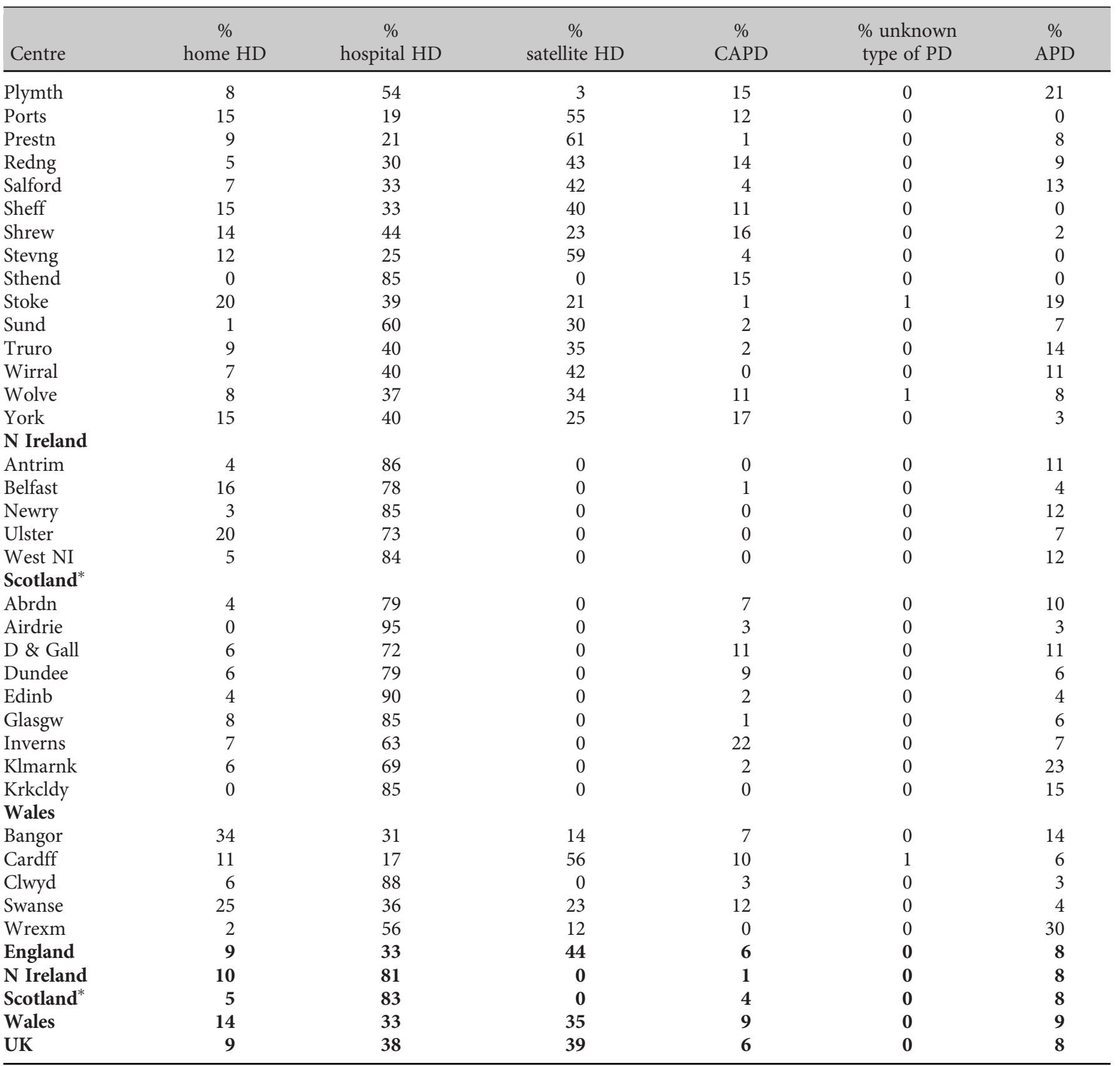

Excluded one centre with $\geqslant 40 \%$ primary renal diagnosis aetiology uncertain (Colchester)

Patients with diabetes as their primary renal disease and patients with a missing primary renal diagnosis code are excluded from this table * All haemodialysis patients in centres in Scotland are shown as receiving treatment at home or in centre as no data is available regarding satellite dialysis 
Table F2.9. Number of 2014 prevalent patients without diabetes aged under 65 by treatment modality

\begin{tabular}{lrrr}
\hline & HD & \multicolumn{1}{c}{ PD } & Transplant \\
\hline England & 6,761 & 1,167 & 17,686 \\
N Ireland & 176 & 17 & 679 \\
Scotland & 665 & 87 & 1,906 \\
Wales & 338 & 71 & 1,009 \\
UK & $\mathbf{7 , 9 4 0}$ & $\mathbf{1 , 3 4 2}$ & $\mathbf{2 1 , 2 8 0}$ \\
\hline
\end{tabular}

Excluded one centre with $\geqslant 40 \%$ primary renal diagnosis aetiology uncertain (Colchester)

Patients with diabetes as their primary renal disease and patients with a missing primary renal diagnosis code are excluded from this table

Table F2.10. Dialysis modalities for 2014 prevalent patients without diabetes aged 65 and over

\begin{tabular}{|c|c|c|c|c|c|c|}
\hline Centre & $\begin{array}{c}\% \\
\text { home HD }\end{array}$ & $\begin{array}{c}\% \\
\text { hospital HD }\end{array}$ & $\begin{array}{c}\% \\
\text { satellite HD }\end{array}$ & $\begin{array}{c}\% \\
\text { CAPD }\end{array}$ & $\begin{array}{l}\% \text { unknown } \\
\text { type of PD }\end{array}$ & $\begin{array}{c}\% \\
\mathrm{APD}\end{array}$ \\
\hline \multicolumn{7}{|l|}{ England } \\
\hline B Heart & 1 & 81 & 12 & 5 & 0 & 1 \\
\hline B QEH & 2 & 9 & 77 & 5 & 0 & 7 \\
\hline Basldn & 0 & 89 & 2 & 6 & 0 & 2 \\
\hline Bradfd & 1 & 58 & 30 & 3 & 0 & 7 \\
\hline Brightn & 5 & 35 & 48 & 10 & 0 & 3 \\
\hline Bristol & 2 & 12 & 77 & 5 & 0 & 5 \\
\hline Camb & 2 & 40 & 50 & 0 & 8 & 0 \\
\hline Carlis & 0 & 48 & 22 & 20 & 0 & 10 \\
\hline Carsh & 2 & 11 & 75 & 3 & 0 & 8 \\
\hline Chelms & 1 & 85 & 0 & 11 & 0 & 3 \\
\hline Covnt & 0 & 85 & 0 & 15 & 0 & 0 \\
\hline Derby & 11 & 69 & 0 & 14 & 0 & 7 \\
\hline Donc & 1 & 44 & 44 & 1 & 0 & 11 \\
\hline Dorset & 1 & 18 & 66 & 5 & 0 & 10 \\
\hline Dudley & 1 & 57 & 26 & 13 & 0 & 3 \\
\hline Exeter & 0 & 10 & 73 & 8 & 0 & 9 \\
\hline Glouc & 1 & 69 & 19 & 3 & 0 & 7 \\
\hline Hull & 1 & 33 & 51 & 7 & 0 & 7 \\
\hline Ipswi & 4 & 62 & 13 & 12 & 0 & 10 \\
\hline Kent & 0 & 26 & 62 & 9 & 0 & 3 \\
\hline L Barts & 0 & 37 & 44 & 5 & 0 & 14 \\
\hline L Guys & 3 & 13 & 81 & 2 & 0 & 2 \\
\hline L Kings & 0 & 14 & 70 & 9 & 0 & 7 \\
\hline L Rfree & 2 & 2 & 81 & 8 & 0 & 6 \\
\hline L St.G & 0 & 32 & 51 & 7 & 1 & 9 \\
\hline L West & 1 & 20 & 74 & 3 & 0 & 2 \\
\hline Leeds & 0 & 13 & 79 & 1 & 0 & 7 \\
\hline Leic & 4 & 15 & 72 & 4 & 0 & 5 \\
\hline Liv Ain & 1 & 7 & 80 & 1 & 0 & 11 \\
\hline Liv Roy & 4 & 32 & 49 & 12 & 0 & 3 \\
\hline M RI & 3 & 16 & 68 & 3 & 0 & 10 \\
\hline
\end{tabular}


Table F2.10. Continued

\begin{tabular}{|c|c|c|c|c|c|c|}
\hline Centre & $\begin{array}{c}\% \\
\text { home HD }\end{array}$ & $\begin{array}{c}\% \\
\text { hospital HD }\end{array}$ & $\begin{array}{c}\% \\
\text { satellite HD }\end{array}$ & $\begin{array}{c}\% \\
\text { CAPD }\end{array}$ & $\begin{array}{l}\% \text { unknown } \\
\text { type of PD }\end{array}$ & $\begin{array}{c}\% \\
\mathrm{APD}\end{array}$ \\
\hline Middlbr & 2 & 17 & 77 & 5 & 0 & 0 \\
\hline Newc & 2 & 83 & 0 & 2 & 0 & 14 \\
\hline Norwch & 6 & 51 & 36 & 5 & 1 & 1 \\
\hline Nottm & 2 & 37 & 46 & 8 & 0 & 7 \\
\hline Oxford & 2 & 32 & 51 & 3 & 0 & 13 \\
\hline Plymth & 3 & 77 & 3 & 4 & 0 & 13 \\
\hline Ports & 1 & 17 & 71 & 11 & 0 & 0 \\
\hline Prestn & 5 & 18 & 67 & 2 & 0 & 9 \\
\hline Redng & 1 & 44 & 42 & 10 & 0 & 3 \\
\hline Salford & 2 & 27 & 51 & 7 & 0 & 13 \\
\hline Sheff & 2 & 36 & 52 & 10 & 0 & 0 \\
\hline Shrew & 3 & 55 & 33 & 6 & 0 & 3 \\
\hline Stevng & 2 & 23 & 68 & 6 & 0 & 0 \\
\hline Sthend & 0 & 87 & 0 & 13 & 0 & 0 \\
\hline Stoke & 2 & 47 & 31 & 4 & 8 & 8 \\
\hline Sund & 0 & 61 & 32 & 3 & 0 & 3 \\
\hline Truro & 2 & 39 & 49 & 8 & 0 & 2 \\
\hline Wirral & 1 & 43 & 50 & 1 & 0 & 5 \\
\hline Wolve & 2 & 35 & 44 & 10 & 3 & 6 \\
\hline York & 0 & 32 & 57 & 9 & 0 & 1 \\
\hline \multicolumn{7}{|l|}{ N Ireland } \\
\hline Antrim & 0 & 92 & 0 & 1 & 0 & 7 \\
\hline Belfast & 1 & 91 & 0 & 1 & 0 & 6 \\
\hline Newry & 2 & 79 & 0 & 0 & 0 & 19 \\
\hline Ulster & 2 & 95 & 0 & 0 & 0 & 3 \\
\hline West NI & 2 & 86 & 0 & 0 & 2 & 11 \\
\hline \multicolumn{7}{|l|}{ Scotland* } \\
\hline Abrdn & 2 & 90 & 0 & 4 & 0 & 3 \\
\hline Airdrie & 0 & 97 & 0 & 1 & 0 & 1 \\
\hline D \& Gall & 0 & 82 & 0 & 18 & 0 & 0 \\
\hline Dundee & 0 & 88 & 0 & 6 & 0 & 5 \\
\hline Edinb & 1 & 89 & 0 & 2 & 0 & 8 \\
\hline Glasgw & 2 & 93 & 0 & 2 & 0 & 4 \\
\hline Inverns & 2 & 89 & 0 & 7 & 0 & 2 \\
\hline Klmarnk & 10 & 73 & 0 & 1 & 0 & 16 \\
\hline Krkcldy & 0 & 93 & 0 & 1 & 0 & 6 \\
\hline \multicolumn{7}{|l|}{ Wales } \\
\hline Bangor & 5 & 47 & 30 & 9 & 0 & 9 \\
\hline Cardff & 2 & 11 & 73 & 9 & 0 & 4 \\
\hline Clwyd & 4 & 79 & 0 & 9 & 0 & 9 \\
\hline Swanse & 4 & 47 & 36 & 10 & 0 & 3 \\
\hline Wrexm & 0 & 67 & 14 & 0 & 0 & 19 \\
\hline England & 2 & 32 & 54 & 6 & $\mathbf{0}$ & 6 \\
\hline N Ireland & 1 & 89 & 0 & 1 & 0 & 9 \\
\hline Scotland ${ }^{*}$ & 2 & 90 & 0 & 3 & 0 & 5 \\
\hline Wales & 3 & 36 & 46 & 9 & $\mathbf{0}$ & 6 \\
\hline UK & 2 & 38 & 48 & 6 & 0 & 6 \\
\hline
\end{tabular}

Excluded one centre with $\geqslant 40 \%$ primary renal diagnosis aetiology uncertain (Colchester)

Patients with diabetes as their primary renal disease and patients with a missing primary renal diagnosis code are excluded from this table *All haemodialysis patients in centres in Scotland are shown as receiving treatment at home or in centre as no data is available regarding satellite dialysis 
Table F2.11. Number of 2014 prevalent patients without diabetes aged 65 and over by treatment modality

\begin{tabular}{lrrr}
\hline & HD & PD & Transplant \\
\hline England & 8,088 & 1,109 & 4,938 \\
N Ireland & 298 & 31 & 157 \\
Scotland & 769 & 72 & 449 \\
Wales & 514 & 85 & 329 \\
UK & $\mathbf{9 , 6 6 9}$ & $\mathbf{1 , 2 9 7}$ & $\mathbf{5 , 8 7 3}$ \\
\hline
\end{tabular}

Excluded one centre with $\geqslant 40 \%$ primary renal diagnosis aetiology uncertain (Colchester)

Patients with diabetes as their primary renal disease and patients with a missing primary renal diagnosis code are excluded from this table

Table F2.12. Dialysis modalities for 2014 prevalent patients with diabetes

\begin{tabular}{|c|c|c|c|c|c|c|}
\hline Centre & $\begin{array}{c}\% \\
\text { home HD }\end{array}$ & $\begin{array}{c}\% \\
\text { hospital HD }\end{array}$ & $\begin{array}{c}\% \\
\text { satellite HD }\end{array}$ & $\begin{array}{c}\% \\
\text { CAPD }\end{array}$ & $\begin{array}{l}\% \text { unknown } \\
\text { type of PD }\end{array}$ & $\begin{array}{c}\% \\
\mathrm{APD}\end{array}$ \\
\hline \multicolumn{7}{|l|}{ England } \\
\hline B QEH & 4 & 13 & 75 & 3 & 0 & 5 \\
\hline Basldn & 0 & 76 & 4 & 7 & 0 & 13 \\
\hline Bradfd & 0 & 86 & 11 & 2 & 0 & 2 \\
\hline Bristol & 2 & 19 & 71 & 3 & 0 & 4 \\
\hline Camb & 5 & 42 & 42 & 0 & 11 & 0 \\
\hline Carlis & 0 & 71 & 18 & 0 & 0 & 12 \\
\hline Carsh & 2 & 17 & 69 & 5 & 1 & 7 \\
\hline Chelms & 0 & 76 & 0 & 9 & 4 & 11 \\
\hline Covnt & 0 & 74 & 0 & 26 & 0 & 0 \\
\hline Exeter & 0 & 10 & 74 & 7 & 0 & 9 \\
\hline Glouc & 0 & 48 & 25 & 0 & 0 & 28 \\
\hline Hull & 1 & 52 & 27 & 6 & 0 & 14 \\
\hline Ipswi & 0 & 60 & 12 & 8 & 0 & 20 \\
\hline Kent & 2 & 27 & 56 & 13 & 0 & 2 \\
\hline L Barts & 0 & 37 & 41 & 4 & 0 & 18 \\
\hline L Guys & 2 & 20 & 76 & 1 & 0 & 1 \\
\hline L Kings & 0 & 20 & 67 & 5 & 0 & 9 \\
\hline L Rfree & 0 & 2 & 80 & 5 & 0 & 12 \\
\hline L St.G & 1 & 37 & 48 & 5 & 0 & 10 \\
\hline
\end{tabular}


Table F2.12. Continued

\begin{tabular}{|c|c|c|c|c|c|c|}
\hline Centre & $\begin{array}{c}\% \\
\text { home HD }\end{array}$ & $\begin{array}{c}\% \\
\text { hospital HD }\end{array}$ & $\begin{array}{c}\% \\
\text { satellite HD }\end{array}$ & $\begin{array}{c}\% \\
\text { CAPD }\end{array}$ & $\begin{array}{l}\% \text { unknown } \\
\text { type of PD }\end{array}$ & $\begin{array}{c}\% \\
\mathrm{APD}\end{array}$ \\
\hline Middlbr & 0 & 28 & 66 & 6 & 0 & 0 \\
\hline Newc & 0 & 81 & 0 & 3 & 0 & 16 \\
\hline Norwch & 6 & 44 & 38 & 12 & 0 & 0 \\
\hline Nottm & 4 & 55 & 24 & 1 & 0 & 16 \\
\hline Oxford & 1 & 35 & 51 & 2 & 0 & 11 \\
\hline Plymth & 0 & 79 & 0 & 11 & 0 & 11 \\
\hline Ports & 4 & 24 & 65 & 7 & 0 & 0 \\
\hline Prestn & 3 & 28 & 60 & 2 & 0 & 6 \\
\hline Redng & 1 & 36 & 36 & 18 & 1 & 8 \\
\hline Salford & 1 & 31 & 55 & 8 & 0 & 5 \\
\hline Sheff & 2 & 46 & 45 & 6 & 0 & 0 \\
\hline Shrew & 2 & 49 & 34 & 12 & 0 & 2 \\
\hline Stevng & 4 & 24 & 68 & 5 & 0 & 0 \\
\hline Sthend & 3 & 80 & 0 & 17 & 0 & 0 \\
\hline Stoke & 8 & 52 & 23 & 2 & 6 & 8 \\
\hline Sund & 0 & 62 & 30 & 4 & 0 & 4 \\
\hline Truro & 9 & 56 & 24 & 6 & 0 & 6 \\
\hline Wirral & 2 & 39 & 43 & 0 & 0 & 15 \\
\hline Wolve & 3 & 39 & 34 & 15 & 7 & 2 \\
\hline York & 6 & 27 & 39 & 18 & 0 & 9 \\
\hline \multicolumn{7}{|l|}{ N Ireland } \\
\hline Antrim & 0 & 89 & 0 & 0 & 0 & 11 \\
\hline Belfast & 0 & 96 & 0 & 0 & 0 & 4 \\
\hline Newry & 0 & 88 & 0 & 0 & 0 & 13 \\
\hline Ulster & 0 & 97 & 0 & 0 & 0 & 3 \\
\hline West NI & 0 & 96 & 0 & 0 & 0 & 4 \\
\hline \multicolumn{7}{|l|}{ Scotland* } \\
\hline Abrdn & 0 & 90 & 0 & 3 & 0 & 7 \\
\hline Airdrie & 0 & 94 & 0 & 4 & 0 & 2 \\
\hline D \& Gall & 5 & 55 & 0 & 25 & 0 & 15 \\
\hline Dundee & 0 & 93 & 0 & 5 & 0 & 2 \\
\hline Edinb & 0 & 92 & 0 & 3 & 0 & 5 \\
\hline Glasgw & 3 & 87 & 0 & 3 & 0 & 7 \\
\hline Inverns & 0 & 77 & 0 & 8 & 0 & 15 \\
\hline Klmarnk & 0 & 83 & 0 & 3 & 0 & 14 \\
\hline Krkcldy & 0 & 89 & 0 & 0 & 0 & 11 \\
\hline \multicolumn{7}{|l|}{ Wales } \\
\hline Bangor & 4 & 46 & 46 & 4 & 0 & 0 \\
\hline Cardff & 6 & 10 & 71 & 9 & 0 & 4 \\
\hline Clwyd & 5 & 84 & 0 & 5 & 0 & 5 \\
\hline Swanse & 7 & 44 & 35 & 11 & 0 & 3 \\
\hline Wrexm & 0 & 85 & 4 & 0 & 0 & 12 \\
\hline England & 2 & 34 & 50 & 6 & 0 & 7 \\
\hline N Ireland & 0 & 93 & 0 & 0 & 0 & 7 \\
\hline Scotland* & 1 & 88 & 0 & 4 & 0 & 7 \\
\hline Wales & 6 & 36 & 46 & 8 & 0 & 4 \\
\hline UK & 2 & 40 & 45 & 5 & 0 & 7 \\
\hline
\end{tabular}

Excluded one centre with $\geqslant 40 \%$ primary renal diagnosis aetiology uncertain (Colchester)

Only patients with diabetes as their primary renal disease included in this table

*All haemodialysis patients in centres in Scotland are shown as receiving treatment at home or in centre as no data is available regarding satellite dialysis 
Table F2.13. Number of 2014 prevalent patients with diabetes by treatment modality

\begin{tabular}{lrrc}
\hline & HD & PD & Transplant \\
\hline England & 4,634 & 692 & 2,686 \\
N Ireland & 158 & 12 & 73 \\
Scotland & 412 & 52 & 254 \\
Wales & 255 & 36 & 192 \\
UK & $\mathbf{5 , 4 5 9}$ & $\mathbf{7 9 2}$ & $\mathbf{3 , 2 0 5}$ \\
\hline
\end{tabular}

Excluded one centre with $\geqslant 40 \%$ primary renal diagnosis aetiology uncertain (Colchester)

Only patients with diabetes as their primary renal disease included in this table

Table F2.14. Demography of 2014 prevalent patients with diabetes

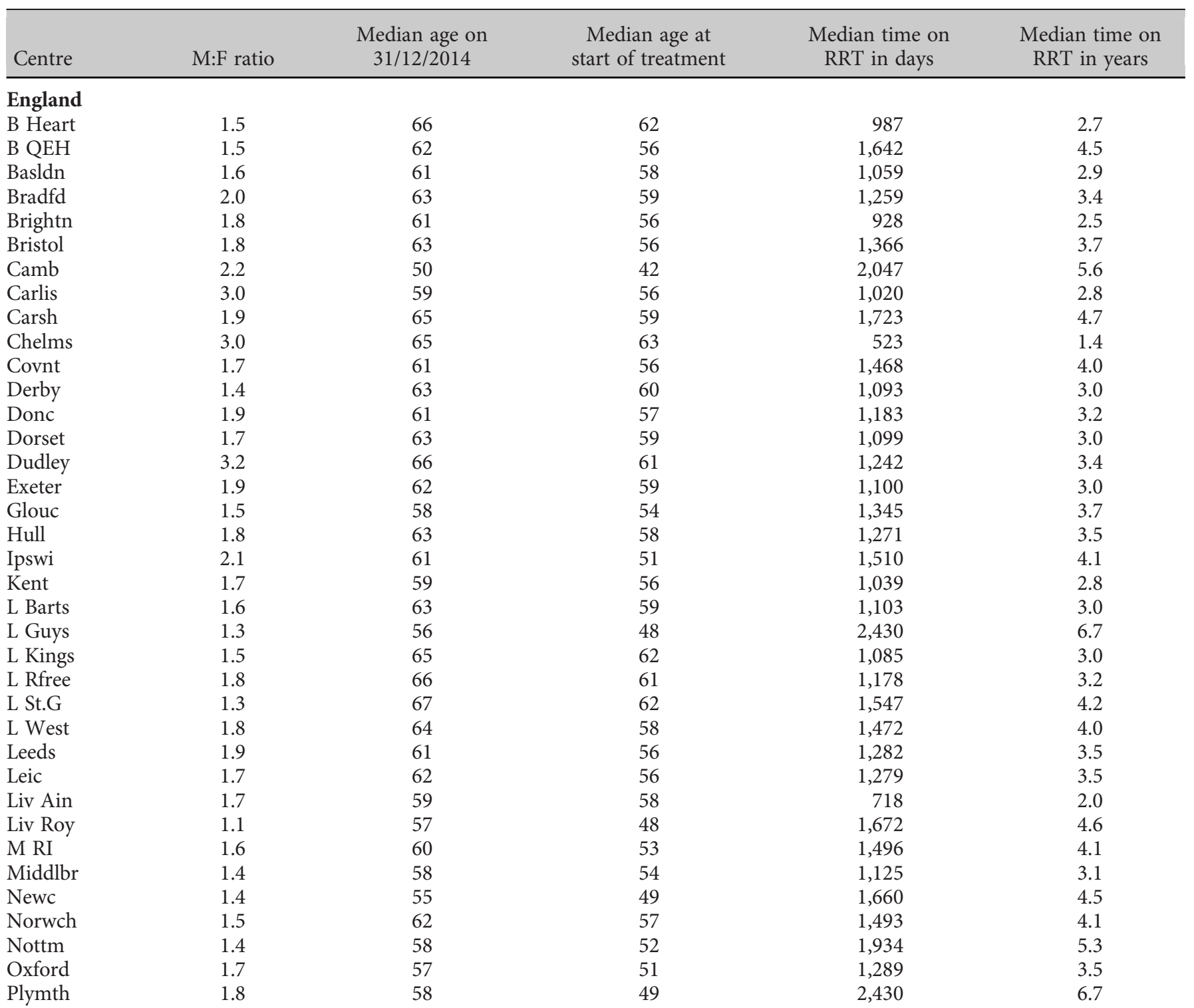


Table F2.14. Continued

\begin{tabular}{|c|c|c|c|c|c|}
\hline Centre & $\mathrm{M}: \mathrm{F}$ ratio & $\begin{array}{c}\text { Median age on } \\
31 / 12 / 2014\end{array}$ & $\begin{array}{c}\text { Median age at } \\
\text { start of treatment }\end{array}$ & $\begin{array}{l}\text { Median time on } \\
\text { RRT in days }\end{array}$ & $\begin{array}{l}\text { Median time on } \\
\text { RRT in years }\end{array}$ \\
\hline Ports & 1.8 & 60 & 57 & 1,166 & 3.2 \\
\hline Redng & 1.7 & 62 & 59 & 1,413 & 3.9 \\
\hline Salford & 2.2 & 62 & 57 & 1,262 & 3.5 \\
\hline Sheff & 2.4 & 61 & 56 & 1,223 & 3.3 \\
\hline Sthend & 1.9 & 62 & 59 & 1,718 & 4.7 \\
\hline Stoke & 1.4 & 65 & 60 & 1,112 & 3.0 \\
\hline Sund & 1.7 & 58 & 56 & 897 & 2.5 \\
\hline Truro & 1.3 & 57 & 52 & 1,422 & 3.9 \\
\hline Wirral & 1.5 & 63 & 56 & 811 & 2.2 \\
\hline Wolve & 1.9 & 60 & 53 & 1,811 & 5.0 \\
\hline Newry & 1.4 & 62 & 59 & 1,378 & 3.8 \\
\hline Ulster & 3.1 & 62 & 57 & 1,158 & 3.2 \\
\hline West NI & 1.6 & 65 & 56 & 1,232 & 3.4 \\
\hline \multicolumn{6}{|l|}{ Scotland } \\
\hline Abrdn & 1.4 & 60 & 54 & 1,224 & 3.4 \\
\hline Airdrie & 1.4 & 59 & 56 & 995 & 2.7 \\
\hline D \& Gall & 1.2 & 63 & 57 & 1,059 & 2.9 \\
\hline Dundee & 1.2 & 58 & 51 & 1,729 & 4.7 \\
\hline Edinb & 1.3 & 53 & 49 & 1,269 & 3.5 \\
\hline Glasgw & 1.3 & 59 & 54 & 1,115 & 3.1 \\
\hline Inverns & 2.0 & 51 & 42 & 2,693 & 7.4 \\
\hline Klmarnk & 1.7 & 56 & 50 & 1,089 & 3.0 \\
\hline England & 1.7 & 62 & 56 & 1,333 & 3.6 \\
\hline N Ireland & 1.7 & 62 & 58 & 1,307 & 3.6 \\
\hline Scotland & 1.3 & 57 & 52 & 1,233 & 3.4 \\
\hline Wales & 1.9 & 61 & 55 & 1,274 & 3.5 \\
\hline UK & 1.7 & 61 & 56 & 1,322 & 3.6 \\
\hline
\end{tabular}

Excluded one centre with $\geqslant 40 \%$ primary renal diagnosis aetiology uncertain (Colchester)

Only patients with diabetes as their primary renal disease included in this table

Table F2.15. Transplant gender ratios in 2014 prevalent patients

\begin{tabular}{lccrrc}
\hline & $\%$ male & \% female & $N$ male & $N$ female & M:F ratio \\
\hline England & 60.6 & 39.4 & 15,812 & 10,293 & 1.5 \\
N Ireland & 61.5 & 38.5 & 561 & 351 & 1.6 \\
Scotland & 59.7 & 40.3 & 1,558 & 1,052 & 1.5 \\
Wales & 62.5 & 37.5 & 960 & 577 & 1.7 \\
UK & $\mathbf{6 0 . 6}$ & $\mathbf{3 9 . 4}$ & $\mathbf{1 8 , 8 9 1}$ & $\mathbf{1 2 , 2 7 3}$ & $\mathbf{1 . 5}$ \\
\hline
\end{tabular}




\section{F:3 Trends by CCG/HB between 2009 and 2014}

Table F3.1. Number of incident patients by year of RRT start and CCG/HB

Blank cells are values of 1 or 2 - these have been suppressed

\begin{tabular}{|c|c|c|c|c|c|c|c|c|}
\hline \multirow[b]{2}{*}{ UK area } & \multirow[b]{2}{*}{ CCG/HB name } & \multirow[b]{2}{*}{ Code } & \multicolumn{6}{|c|}{ Incident numbers } \\
\hline & & & 2009 & 2010 & 2011 & 2012 & 2013 & 2014 \\
\hline \multirow{6}{*}{$\begin{array}{l}\text { Cheshire, Warrington } \\
\text { and Wirral }\end{array}$} & NHS Eastern Cheshire & E38000056 & 18 & 20 & 18 & 17 & 16 & 20 \\
\hline & NHS South Cheshire & E38000151 & 14 & 14 & 15 & 12 & 24 & 22 \\
\hline & NHS Vale Royal & E38000189 & 10 & 9 & 10 & 9 & 15 & \\
\hline & NHS Warrington & E38000194 & 22 & 13 & 10 & 19 & 16 & 24 \\
\hline & NHS West Cheshire & E38000196 & 24 & 31 & 28 & 22 & 26 & 25 \\
\hline & NHS Wirral & E38000208 & 30 & 32 & 35 & 22 & 38 & 27 \\
\hline \multirow{5}{*}{$\begin{array}{l}\text { Durham, Darlington } \\
\text { and Tees }\end{array}$} & NHS Darlington & E38000042 & 11 & 11 & 11 & 15 & 10 & 7 \\
\hline & NHS Durham Dales, Easington and Sedgefield & E38000047 & 31 & 32 & 35 & 27 & 33 & 33 \\
\hline & NHS Hartlepool and Stockton-on-Tees & E38000075 & 21 & 24 & 28 & 33 & 27 & 31 \\
\hline & NHS North Durham & E38000116 & 14 & 13 & 15 & 35 & 18 & 16 \\
\hline & NHS South Tees & E38000162 & 23 & 31 & 28 & 29 & 37 & 27 \\
\hline \multirow[t]{12}{*}{ Greater Manchester } & NHS Bolton & E38000016 & 24 & 39 & 27 & 26 & 26 & 20 \\
\hline & NHS Bury & E38000024 & 16 & 13 & 14 & 27 & 16 & 24 \\
\hline & NHS Central Manchester & E38000032 & 22 & 25 & 14 & 21 & 28 & 31 \\
\hline & NHS Heywood, Middleton \& Rochdale & E38000080 & 24 & 16 & 26 & 27 & 26 & 29 \\
\hline & NHS North Manchester & E38000123 & 22 & 12 & 20 & 20 & 20 & 21 \\
\hline & NHS Oldham & E38000135 & 19 & 18 & 23 & 16 & 22 & 30 \\
\hline & NHS Salford & E38000143 & 22 & 30 & 17 & 20 & 26 & 20 \\
\hline & NHS South Manchester & E38000158 & 11 & 13 & 16 & 16 & 17 & 13 \\
\hline & NHS Stockport & E38000174 & 17 & 29 & 28 & 21 & 17 & 31 \\
\hline & NHS Tameside and Glossop & E38000182 & 23 & 24 & 26 & 16 & 30 & 25 \\
\hline & NHS Trafford & E38000187 & 26 & 30 & 12 & 28 & 28 & 22 \\
\hline & NHS Wigan Borough & E38000205 & 20 & 25 & 35 & 27 & 26 & 33 \\
\hline \multirow[t]{8}{*}{ Lancashire } & NHS Blackburn with Darwen & E38000014 & 12 & 13 & 20 & 17 & 13 & 11 \\
\hline & NHS Blackpool & E38000015 & 16 & 10 & 14 & 24 & 19 & 20 \\
\hline & NHS Chorley and South Ribble & E38000034 & 24 & 10 & 18 & 14 & 25 & 18 \\
\hline & NHS East Lancashire & E38000050 & 33 & 29 & 37 & 22 & 36 & 47 \\
\hline & NHS Fylde \& Wyre & $\mathrm{E} 38000060$ & 19 & 15 & 12 & 17 & 18 & 23 \\
\hline & NHS Greater Preston & E38000065 & 14 & 11 & 11 & 21 & 18 & 20 \\
\hline & NHS Lancashire North & E38000093 & 11 & 10 & 18 & 12 & 11 & 12 \\
\hline & NHS West Lancashire & E38000200 & 8 & 7 & 11 & 10 & 9 & 9 \\
\hline \multirow[t]{6}{*}{ Merseyside } & NHS Halton & E38000068 & 14 & 11 & 20 & 13 & 13 & 15 \\
\hline & NHS Knowsley & E38000091 & 11 & 13 & 17 & 20 & 11 & 28 \\
\hline & NHS Liverpool & E38000101 & 54 & 38 & 50 & 55 & 47 & 59 \\
\hline & NHS South Sefton & E38000161 & 14 & 23 & 25 & 19 & 24 & 27 \\
\hline & NHS Southport and Formby & E38000170 & 12 & 9 & 14 & 11 & 21 & 14 \\
\hline & NHS St Helens & E38000172 & 14 & 18 & 15 & 18 & 13 & 21 \\
\hline \multirow{8}{*}{$\begin{array}{l}\text { Cumbria, Northumberland, } \\
\text { Tyne and Wear }\end{array}$} & NHS Cumbria & E38000041 & 38 & 45 & 36 & 39 & 59 & 55 \\
\hline & NHS Gateshead & E38000061 & 20 & 17 & 17 & 20 & 12 & 17 \\
\hline & NHS Newcastle North and East & E38000111 & 13 & 11 & 11 & 9 & 6 & 11 \\
\hline & NHS Newcastle West & E38000112 & 12 & 9 & 12 & 12 & 13 & 17 \\
\hline & NHS North Tyneside & E38000127 & 20 & 20 & 15 & 20 & 22 & 16 \\
\hline & NHS Northumberland & E38000130 & 24 & 23 & 32 & 30 & 25 & 38 \\
\hline & NHS South Tyneside & E38000163 & 22 & 12 & 18 & 9 & 13 & 11 \\
\hline & NHS Sunderland & E38000176 & 29 & 31 & 23 & 27 & 19 & 30 \\
\hline
\end{tabular}


Table F3.1. Continued

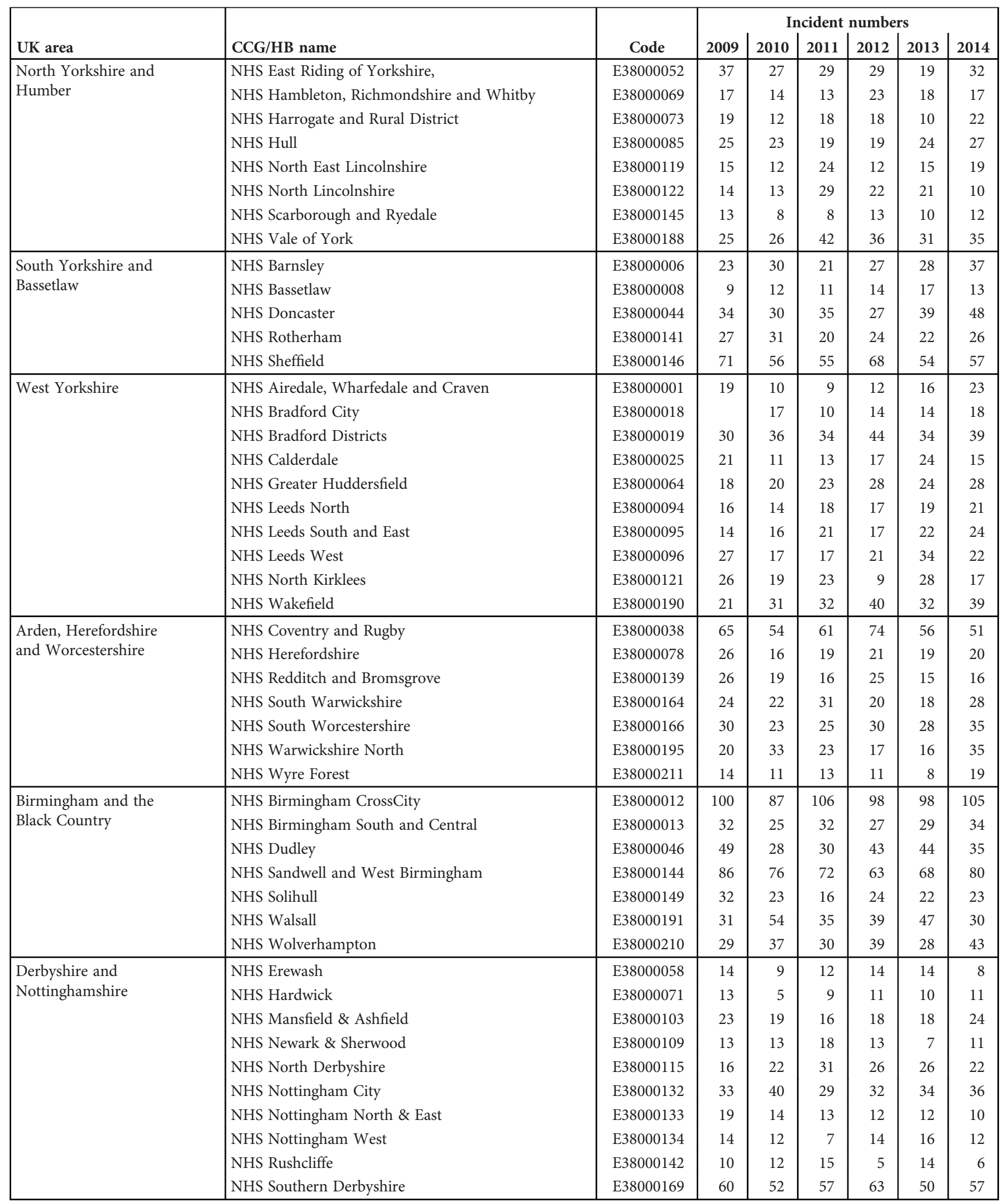


Table F3.1. Continued

\begin{tabular}{|c|c|c|c|c|c|c|c|c|}
\hline \multirow[b]{2}{*}{ UK area } & \multirow[b]{2}{*}{ CCG/HB name } & \multirow[b]{2}{*}{ Code } & \multicolumn{6}{|c|}{ Incident numbers } \\
\hline & & & 2009 & 2010 & 2011 & 2012 & 2013 & 2014 \\
\hline \multirow[t]{6}{*}{ East Anglia } & NHS Cambridgeshire and Peterborough & E38000026 & 94 & 67 & 81 & 60 & 98 & 77 \\
\hline & NHS Ipswich and East Suffolk & E38000086 & 39 & 31 & 29 & 42 & 44 & 36 \\
\hline & NHS North Norfolk & E38000124 & 11 & 18 & 12 & 18 & 20 & 22 \\
\hline & NHS Norwich & E38000131 & 24 & 23 & 23 & 18 & 16 & 20 \\
\hline & NHS West Norfolk & E38000203 & 15 & 18 & 14 & 15 & 14 & 21 \\
\hline & NHS West Suffolk & E38000204 & 22 & 21 & 18 & 23 & 22 & 17 \\
\hline \multirow[t]{3}{*}{ Essex } & NHS Basildon and Brentwood & E38000007 & 24 & 23 & 28 & 34 & 25 & 30 \\
\hline & NHS Castle Point, Rayleigh and Rochford & $\mathrm{E} 38000030$ & 12 & 18 & 16 & 15 & 26 & 17 \\
\hline & NHS Mid Essex & E38000106 & 37 & 35 & 42 & 35 & 32 & 38 \\
\hline \multirow{7}{*}{$\begin{array}{l}\text { Hertfordshire and the } \\
\text { South Midlands }\end{array}$} & NHS Bedfordshire & E38000010 & 38 & 38 & 33 & 44 & 47 & 47 \\
\hline & NHS Corby & E38000037 & 8 & 8 & 7 & 5 & 4 & 7 \\
\hline & NHS East and North Hertfordshire & E38000049 & 39 & 49 & 60 & 40 & 64 & 65 \\
\hline & NHS Herts Valleys & E38000079 & 54 & 48 & 46 & 52 & 55 & 70 \\
\hline & NHS Luton & E38000102 & 19 & 19 & 25 & 22 & 37 & 30 \\
\hline & NHS Milton Keynes & E38000107 & 21 & 24 & 23 & 27 & 22 & 31 \\
\hline & NHS Nene & E38000108 & 53 & 48 & 59 & 72 & 67 & 69 \\
\hline \multirow{3}{*}{$\begin{array}{l}\text { Leicestershire and } \\
\text { Lincolnshire }\end{array}$} & NHS East Leicestershire and Rutland & E38000051 & 20 & 26 & 27 & 37 & 35 & 32 \\
\hline & NHS Leicester City & E38000097 & 42 & 47 & 51 & 46 & 50 & 37 \\
\hline & NHS Lincolnshire East & E38000099 & 21 & 23 & 27 & 23 & 34 & 19 \\
\hline \multirow{6}{*}{$\begin{array}{l}\text { Shropshire and } \\
\text { Staffordshire }\end{array}$} & NHS North Staffordshire & E38000126 & 28 & 17 & 28 & 15 & 23 & 28 \\
\hline & NHS Shropshire & E38000147 & 26 & 34 & 37 & 29 & 40 & 37 \\
\hline & NHS South East Staffs and Seisdon and Peninsular & E38000153 & 21 & 18 & 26 & 19 & 17 & 22 \\
\hline & NHS Stafford and Surrounds & E38000173 & 20 & 20 & 15 & 17 & 17 & 17 \\
\hline & NHS Stoke on Trent & E38000175 & 37 & 36 & 28 & 23 & 31 & 42 \\
\hline & NHS Telford \& Wrekin & E38000183 & 21 & 23 & 19 & 21 & 22 & 24 \\
\hline \multirow[t]{11}{*}{ London } & NHS Barking \& Dagenham & E38000004 & 21 & 20 & 25 & 31 & 25 & 34 \\
\hline & NHS Barnet & E38000005 & 42 & 58 & 49 & 52 & 44 & 50 \\
\hline & NHS Camden & E38000027 & 27 & 32 & 23 & 24 & 28 & 26 \\
\hline & NHS City and Hackney & E38000035 & 36 & 30 & 34 & 41 & 38 & 47 \\
\hline & NHS Enfield & E38000057 & 38 & 38 & 57 & 47 & 48 & 49 \\
\hline & NHS Haringey & E38000072 & 21 & 30 & 37 & 50 & 50 & 39 \\
\hline & NHS Havering & E38000077 & 18 & 9 & 31 & 28 & 22 & 27 \\
\hline & NHS Islington & E38000088 & 25 & 25 & 27 & 36 & 27 & 21 \\
\hline & NHS Newham & E38000113 & 46 & 50 & 50 & 45 & 52 & 59 \\
\hline & NHS Redbridge & E38000138 & 44 & 37 & 35 & 55 & 53 & 41 \\
\hline & NHS Tower Hamlets & E38000186 & 31 & 26 & 32 & 37 & 41 & 47 \\
\hline
\end{tabular}


Table F3.1. Continued

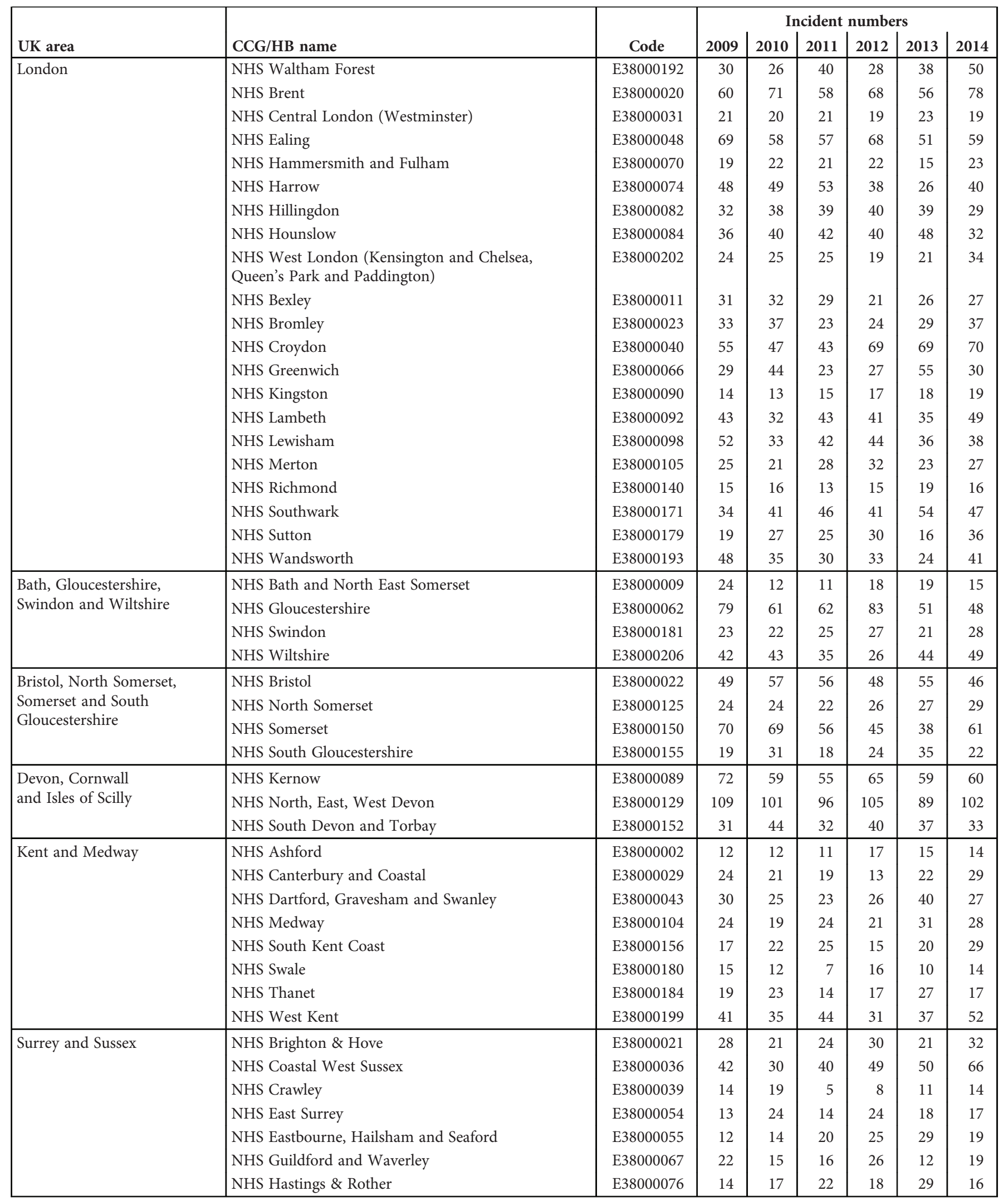


Table F3.1. Continued

\begin{tabular}{|c|c|c|c|c|c|c|c|c|}
\hline \multirow[b]{2}{*}{ UK area } & \multirow[b]{2}{*}{ CCG/HB name } & \multirow[b]{2}{*}{ Code } & \multicolumn{6}{|c|}{ Incident numbers } \\
\hline & & & 2009 & 2010 & 2011 & 2012 & 2013 & 2014 \\
\hline \multirow[t]{5}{*}{ Surrey and Sussex } & NHS High Weald Lewes Havens & E38000081 & 15 & 13 & 14 & 19 & 13 & 22 \\
\hline & NHS Horsham and Mid Sussex & E38000083 & 19 & 18 & 20 & 13 & 20 & 23 \\
\hline & NHS North West Surrey & E38000128 & 30 & 40 & 47 & 33 & 35 & 48 \\
\hline & NHS Surrey Downs & E38000177 & 35 & 30 & 31 & 28 & 35 & 34 \\
\hline & NHS Surrey Heath & E38000178 & 12 & 8 & 8 & 8 & 5 & 5 \\
\hline \multirow[t]{10}{*}{ Thames Valley } & NHS Aylesbury Vale & E38000003 & 12 & 20 & 22 & 16 & 15 & 18 \\
\hline & NHS Bracknell and Ascot & E38000017 & 10 & 13 & 10 & 5 & 16 & 14 \\
\hline & NHS Chiltern & E38000033 & 40 & 23 & 24 & 26 & 35 & 30 \\
\hline & NHS Newbury and District & E38000110 & 12 & 7 & 7 & 8 & 13 & 11 \\
\hline & NHS North \& West Reading & E38000114 & 3 & 3 & 10 & 10 & 7 & 12 \\
\hline & NHS Oxfordshire & E38000136 & 68 & 59 & 69 & 67 & 62 & 62 \\
\hline & NHS Slough & E38000148 & 21 & 22 & 25 & 20 & 21 & 21 \\
\hline & NHS South Reading & E38000160 & 11 & 11 & 10 & 10 & 21 & 15 \\
\hline & NHS Windsor, Ascot and Maidenhead & E38000207 & 17 & 13 & 18 & 9 & 20 & 19 \\
\hline & NHS Wokingham & E38000209 & 13 & 13 & 22 & 8 & 14 & 14 \\
\hline \multirow[t]{9}{*}{ Wessex } & NHS Dorset & E38000045 & 59 & 56 & 68 & 67 & 69 & 73 \\
\hline & NHS Fareham and Gosport & E38000059 & 25 & 25 & 18 & 18 & 24 & 27 \\
\hline & NHS Isle of Wight & E38000087 & & 11 & 14 & 16 & 24 & 17 \\
\hline & NHS North East Hampshire and Farnham & E38000118 & 19 & 18 & 18 & 25 & 26 & 21 \\
\hline & NHS North Hampshire & E38000120 & 12 & 16 & 16 & 11 & 17 & 25 \\
\hline & NHS Portsmouth & E38000137 & 12 & 10 & 25 & 21 & 22 & 19 \\
\hline & NHS South Eastern Hampshire & E38000154 & 26 & 26 & 19 & 16 & 25 & 30 \\
\hline & NHS Southampton & E38000167 & 17 & 26 & 25 & 19 & 14 & 23 \\
\hline & NHS West Hampshire & E38000198 & 43 & 30 & 44 & 41 & 45 & 54 \\
\hline \multirow[t]{7}{*}{ Wales } & Betsi Cadwaladr University & W11000023 & 78 & 78 & 68 & 83 & 77 & 95 \\
\hline & Powys Teaching & W11000024 & 18 & 12 & 22 & 22 & 13 & 11 \\
\hline & Hywel Dda & W11000025 & 36 & 51 & 58 & 42 & 52 & 58 \\
\hline & Abertawe Bro Morgannwg University & W11000026 & 88 & 85 & 68 & 84 & 63 & 47 \\
\hline & Cwm Taf & W11000027 & 40 & 31 & 46 & 29 & 37 & 39 \\
\hline & Aneurin Bevan & W11000028 & 61 & 81 & 77 & 76 & 69 & 82 \\
\hline & Cardiff and Vale University & W11000029 & 53 & 59 & 47 & 47 & 53 & 46 \\
\hline \multirow[t]{14}{*}{ Scotland } & Ayrshire and Arran & S08000015 & 39 & 48 & 36 & 42 & 45 & 40 \\
\hline & Borders & S08000016 & 13 & 15 & 8 & 8 & 7 & 9 \\
\hline & Dumfries and Galloway & S08000017 & 21 & 11 & 11 & 20 & 9 & 24 \\
\hline & Fife & S08000018 & 50 & 50 & 48 & 36 & 43 & 43 \\
\hline & Forth Valley & S08000019 & 32 & 33 & 27 & 28 & 34 & 33 \\
\hline & Grampian & S08000020 & 52 & 51 & 51 & 52 & 58 & 51 \\
\hline & $\begin{array}{l}\text { Greater } \\
\text { Glasgow and Clyde }\end{array}$ & S08000021 & 120 & 103 & 129 & 133 & 111 & 122 \\
\hline & Highland & S08000022 & 28 & 24 & 20 & 24 & 27 & 23 \\
\hline & Lanarkshire & S08000023 & 57 & 64 & 58 & 76 & 68 & 69 \\
\hline & Lothian & S08000024 & 71 & 52 & 62 & 65 & 54 & 71 \\
\hline & Orkney & S08000025 & 3 & & 0 & 5 & & 0 \\
\hline & Shetland & S08000026 & & & & 0 & & 3 \\
\hline & Tayside & S08000027 & 59 & 47 & 56 & 32 & 42 & 48 \\
\hline & Western Isles & S08000028 & 3 & 5 & 0 & 0 & 4 & 6 \\
\hline \multirow[t]{5}{*}{ Northern Ireland } & Belfast & ZC010 & 26 & 43 & 36 & 56 & 40 & 30 \\
\hline & Northern & $\mathrm{ZC} 020$ & 39 & 50 & 59 & 54 & 51 & 52 \\
\hline & Southern & $\mathrm{ZC} 030$ & 26 & 34 & 44 & 28 & 30 & 28 \\
\hline & South Eastern & $\mathrm{ZC} 040$ & 24 & 25 & 34 & 29 & 35 & 29 \\
\hline & Western & $\mathrm{ZC} 050$ & 35 & 24 & 28 & 17 & 29 & 33 \\
\hline
\end{tabular}


Table F3.2 Number of prevalent patients on HD in-centre by year and CCG/HB

\begin{tabular}{|c|c|c|c|c|c|c|c|c|}
\hline \multirow[b]{2}{*}{ UK area } & \multirow[b]{2}{*}{ CCG/HB name } & \multirow[b]{2}{*}{ Code } & \multicolumn{6}{|c|}{ Prevalent numbers on HD in-centre } \\
\hline & & & 2009 & 2010 & 2011 & 2012 & 2013 & 2014 \\
\hline \multirow{6}{*}{$\begin{array}{l}\text { Cheshire, Warrington } \\
\text { and Wirral }\end{array}$} & NHS Eastern Cheshire & E38000056 & 35 & 45 & 43 & 51 & 49 & 51 \\
\hline & NHS South Cheshire & E38000151 & 60 & 58 & 59 & 53 & 52 & 56 \\
\hline & NHS Vale Royal & E38000189 & 23 & 23 & 26 & 23 & 29 & 27 \\
\hline & NHS Warrington & E38000194 & 55 & 53 & 48 & 50 & 49 & 59 \\
\hline & NHS West Cheshire & E38000196 & 83 & 84 & 90 & 83 & 83 & 84 \\
\hline & NHS Wirral & E38000208 & 94 & 97 & 95 & 105 & 111 & 102 \\
\hline \multirow{5}{*}{$\begin{array}{l}\text { Durham, Darlington } \\
\text { and Tees }\end{array}$} & NHS Darlington & E38000042 & 36 & 33 & 25 & 32 & 29 & 27 \\
\hline & NHS Durham Dales, Easington and Sedgefield & E38000047 & 92 & 93 & 102 & 98 & 107 & 104 \\
\hline & NHS Hartlepool and Stockton-on-Tees & E38000075 & 67 & 64 & 83 & 92 & 90 & 96 \\
\hline & NHS North Durham & E38000116 & 51 & 44 & 49 & 65 & 68 & 69 \\
\hline & NHS South Tees & E38000162 & 83 & 85 & 91 & 91 & 100 & 96 \\
\hline \multirow[t]{12}{*}{ Greater Manchester } & NHS Bolton & E38000016 & 60 & 72 & 78 & 72 & 72 & 67 \\
\hline & NHS Bury & E38000024 & 38 & 42 & 44 & 43 & 49 & 53 \\
\hline & NHS Central Manchester & E38000032 & 71 & 78 & 76 & 84 & 89 & 97 \\
\hline & NHS Heywood, Middleton \& Rochdale & E38000080 & 61 & 53 & 52 & 56 & 66 & 74 \\
\hline & NHS North Manchester & E38000123 & 56 & 54 & 59 & 60 & 53 & 56 \\
\hline & NHS Oldham & E38000135 & 58 & 53 & 52 & 56 & 60 & 66 \\
\hline & NHS Salford & E38000143 & 50 & 55 & 52 & 53 & 56 & 55 \\
\hline & NHS South Manchester & E38000158 & 45 & 46 & 42 & 42 & 47 & 45 \\
\hline & NHS Stockport & E38000174 & 48 & 58 & 66 & 69 & 57 & 70 \\
\hline & NHS Tameside and Glossop & E38000182 & 63 & 62 & 54 & 62 & 62 & 60 \\
\hline & NHS Trafford & E38000187 & 50 & 57 & 52 & 55 & 61 & 63 \\
\hline & NHS Wigan Borough & E38000205 & 77 & 68 & 75 & 81 & 84 & 94 \\
\hline \multirow[t]{8}{*}{ Lancashire } & NHS Blackburn with Darwen & E38000014 & 67 & 71 & 71 & 73 & 78 & 74 \\
\hline & NHS Blackpool & E38000015 & 40 & 38 & 41 & 49 & 56 & 63 \\
\hline & NHS Chorley and South Ribble & E38000034 & 40 & 39 & 41 & 54 & 63 & 61 \\
\hline & NHS East Lancashire & E38000050 & 118 & 121 & 116 & 112 & 121 & 128 \\
\hline & NHS Fylde \& Wyre & E38000060 & 59 & 61 & 65 & 66 & 63 & 68 \\
\hline & NHS Greater Preston & E38000065 & 62 & 68 & 65 & 62 & 64 & 57 \\
\hline & NHS Lancashire North & E38000093 & 29 & 31 & 36 & 32 & 26 & 34 \\
\hline & NHS West Lancashire & E38000200 & 37 & 39 & 37 & 35 & 30 & 30 \\
\hline \multirow[t]{6}{*}{ Merseyside } & NHS Halton & E38000068 & 37 & 38 & 47 & 40 & 42 & 44 \\
\hline & NHS Knowsley & E38000091 & 51 & 44 & 51 & 51 & 46 & 48 \\
\hline & NHS Liverpool & E38000101 & 177 & 173 & 174 & 166 & 159 & 158 \\
\hline & NHS South Sefton & E38000161 & 46 & 45 & 61 & 53 & 52 & 61 \\
\hline & NHS Southport and Formby & E38000170 & 41 & 39 & 45 & 44 & 41 & 46 \\
\hline & NHS St Helens & E38000172 & 59 & 61 & 61 & 58 & 50 & 48 \\
\hline \multirow{8}{*}{$\begin{array}{l}\text { Cumbria, Northumberland, } \\
\text { Tyne and Wear }\end{array}$} & NHS Cumbria & E38000041 & 102 & 103 & 100 & 103 & 111 & 116 \\
\hline & NHS Gateshead & E38000061 & 52 & 53 & 44 & 47 & 42 & 47 \\
\hline & NHS Newcastle North and East & E38000111 & 28 & 28 & 30 & 30 & 31 & 28 \\
\hline & NHS Newcastle West & E38000112 & 45 & 43 & 38 & 43 & 39 & 39 \\
\hline & NHS North Tyneside & E38000127 & 39 & 38 & 34 & 39 & 53 & 53 \\
\hline & NHS Northumberland & E38000130 & 71 & 69 & 70 & 69 & 61 & 75 \\
\hline & NHS South Tyneside & E38000163 & 50 & 43 & 49 & 46 & 43 & 40 \\
\hline & NHS Sunderland & E38000176 & 87 & 92 & 88 & 94 & 84 & 95 \\
\hline
\end{tabular}


Table F3.2 Continued

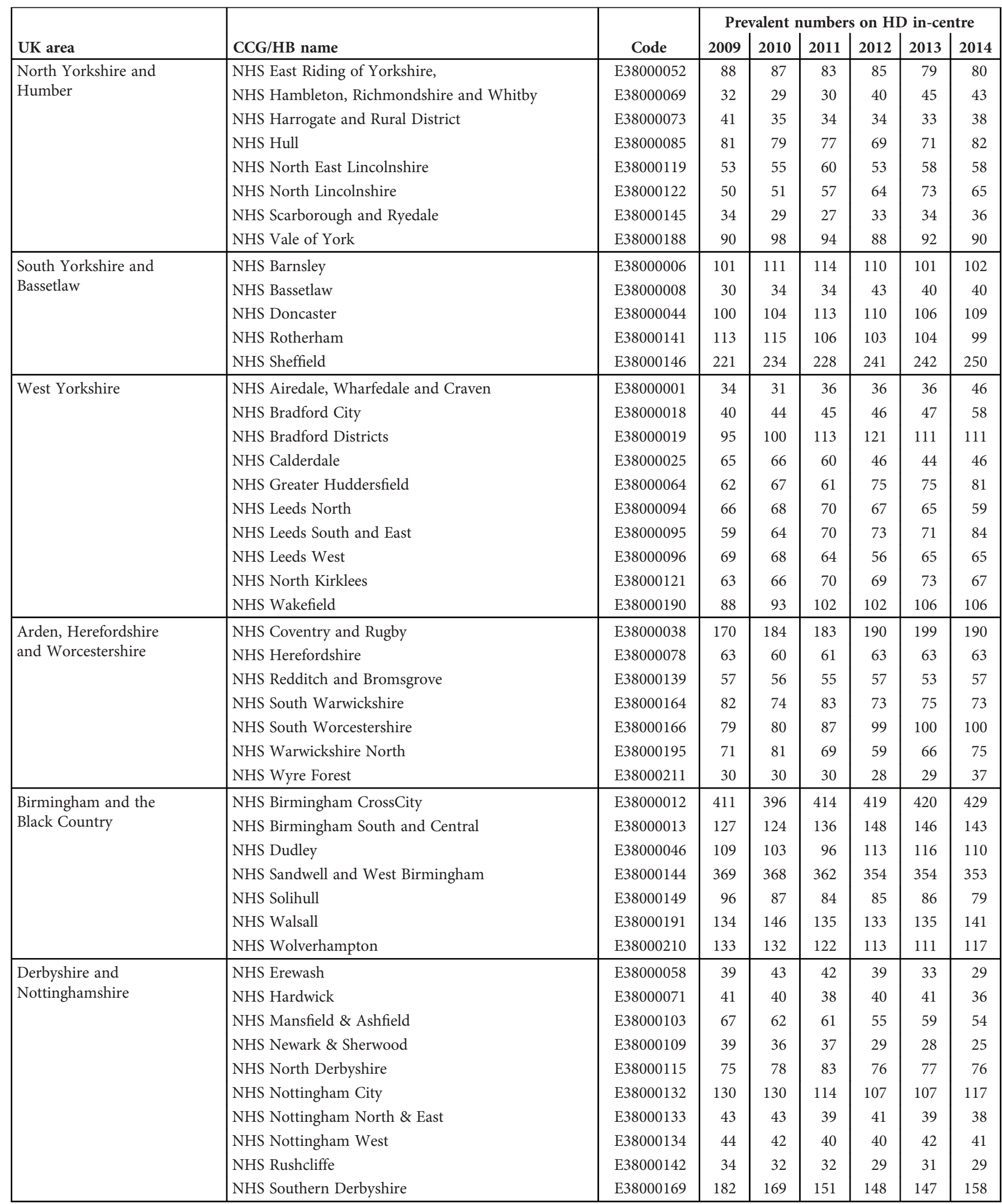


Table F3.2 Continued

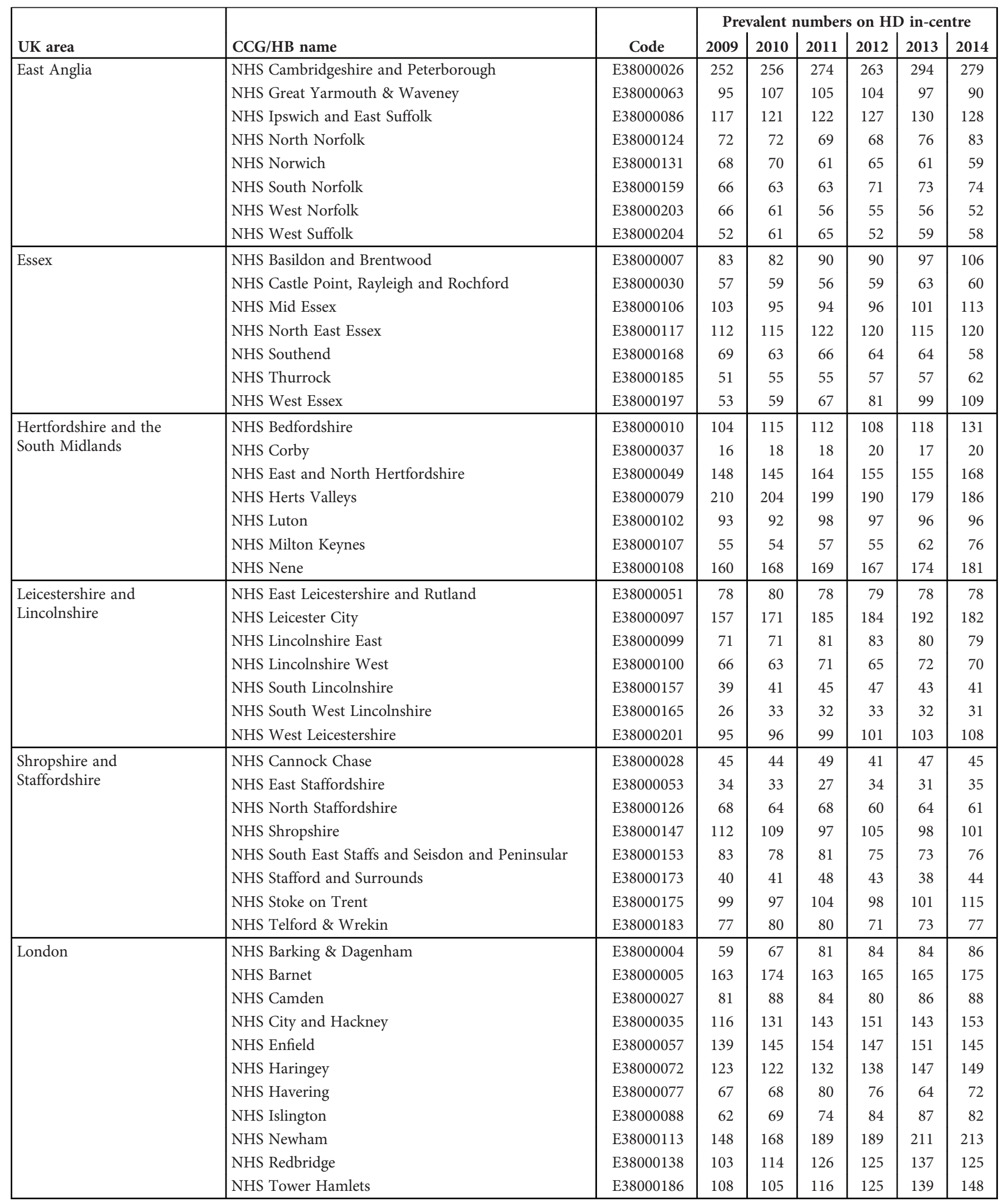


Table F3.2 Continued

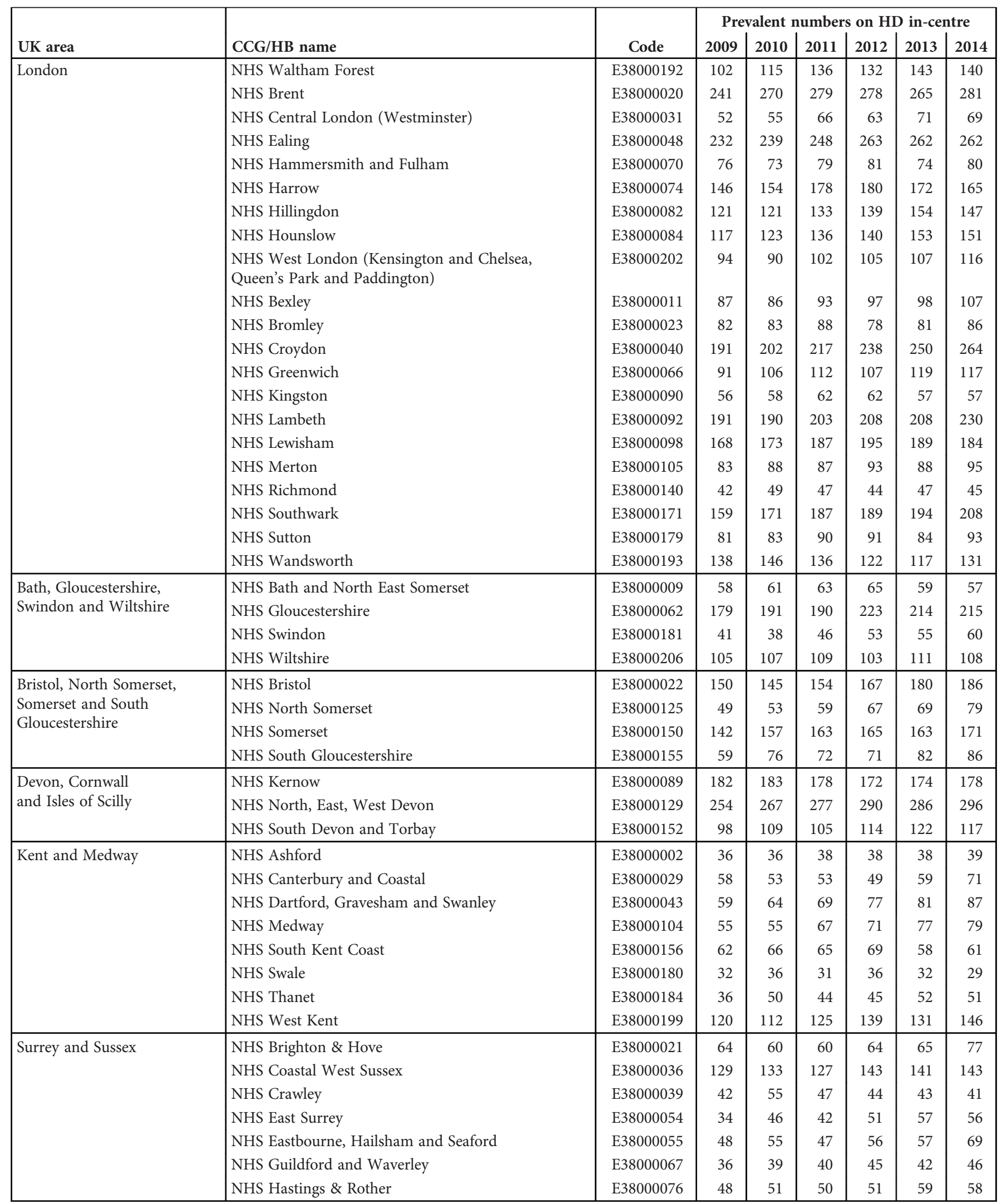


Table F3.2 Continued

\begin{tabular}{|c|c|c|c|c|c|c|c|c|}
\hline \multirow[b]{2}{*}{ UK area } & \multirow[b]{2}{*}{ CCG/HB name } & \multirow[b]{2}{*}{ Code } & \multicolumn{6}{|c|}{ Prevalent numbers on HD in-centre } \\
\hline & & & 2009 & 2010 & 2011 & 2012 & 2013 & 2014 \\
\hline \multirow[t]{5}{*}{ Surrey and Sussex } & NHS High Weald Lewes Havens & E38000081 & 34 & 32 & 33 & 38 & 41 & 46 \\
\hline & NHS Horsham and Mid Sussex & E38000083 & 49 & 44 & 53 & 53 & 55 & 45 \\
\hline & NHS North West Surrey & E38000128 & 106 & 110 & 109 & 107 & 105 & 115 \\
\hline & NHS Surrey Downs & E38000177 & 81 & 82 & 86 & 85 & 91 & 87 \\
\hline & NHS Surrey Heath & E38000178 & 26 & 28 & 26 & 24 & 23 & 21 \\
\hline \multirow[t]{10}{*}{ Thames Valley } & NHS Aylesbury Vale & E38000003 & 48 & 49 & 42 & 50 & 47 & 51 \\
\hline & NHS Bracknell and Ascot & E38000017 & 25 & 25 & 24 & 25 & 32 & 38 \\
\hline & NHS Chiltern & E38000033 & 69 & 66 & 79 & 74 & 81 & 86 \\
\hline & NHS Newbury and District & E38000110 & 18 & 20 & 20 & 14 & 23 & 30 \\
\hline & NHS North \& West Reading & E38000114 & 25 & 20 & 20 & 21 & 21 & 25 \\
\hline & NHS Oxfordshire & E38000136 & 129 & 139 & 160 & 155 & 153 & 154 \\
\hline & NHS Slough & E38000148 & 55 & 59 & 71 & 72 & 64 & 68 \\
\hline & NHS South Reading & E38000160 & 37 & 36 & 32 & 31 & 38 & 39 \\
\hline & NHS Windsor, Ascot and Maidenhead & E38000207 & 42 & 41 & 36 & 38 & 38 & 39 \\
\hline & NHS Wokingham & E38000209 & 45 & 39 & 47 & 43 & 45 & 41 \\
\hline \multirow[t]{9}{*}{ Wessex } & NHS Dorset & E38000045 & 198 & 204 & 207 & 227 & 226 & 234 \\
\hline & NHS Fareham and Gosport & E38000059 & 43 & 45 & 49 & 47 & 58 & 56 \\
\hline & NHS Isle of Wight & E38000087 & 18 & 21 & 29 & 38 & 55 & 56 \\
\hline & NHS North East Hampshire and Farnham & E38000118 & 53 & 51 & 57 & 61 & 66 & 61 \\
\hline & NHS North Hampshire & E38000120 & 40 & 45 & 42 & 41 & 38 & 49 \\
\hline & NHS Portsmouth & E38000137 & 55 & 47 & 56 & 60 & 63 & 64 \\
\hline & NHS South Eastern Hampshire & E38000154 & 60 & 65 & 69 & 60 & 68 & 70 \\
\hline & NHS Southampton & E38000167 & 58 & 68 & 71 & 76 & 69 & 60 \\
\hline & NHS West Hampshire & E38000198 & 121 & 108 & 125 & 134 & 136 & 143 \\
\hline \multirow[t]{7}{*}{ Wales } & Betsi Cadwaladr University & W11000023 & 223 & 220 & 218 & 242 & 230 & 255 \\
\hline & Powys Teaching & W11000024 & 52 & 47 & 43 & 53 & 53 & 46 \\
\hline & Hywel Dda & W11000025 & 133 & 132 & 130 & 122 & 128 & 134 \\
\hline & Abertawe Bro Morgannwg University & W11000026 & 223 & 233 & 221 & 206 & 201 & 178 \\
\hline & Cwm Taf & W11000027 & 109 & 90 & 102 & 96 & 91 & 94 \\
\hline & Aneurin Bevan & W11000028 & 178 & 186 & 194 & 177 & 181 & 204 \\
\hline & Cardiff and Vale University & W11000029 & 137 & 139 & 132 & 132 & 137 & 129 \\
\hline \multirow[t]{14}{*}{ Scotland } & Ayrshire and Arran & S08000015 & 144 & 151 & 143 & 141 & 130 & 126 \\
\hline & Borders & S08000016 & 49 & 50 & 46 & 38 & 35 & 35 \\
\hline & Dumfries and Galloway & S08000017 & 51 & 58 & 51 & 53 & 46 & 46 \\
\hline & Fife & S08000018 & 130 & 138 & 153 & 158 & 153 & 145 \\
\hline & Forth Valley & S08000019 & 107 & 117 & 109 & 99 & 101 & 92 \\
\hline & Grampian & S08000020 & 172 & 181 & 196 & 216 & 203 & 185 \\
\hline & Greater Glasgow and Clyde & S08000021 & 395 & 398 & 409 & 406 & 388 & 371 \\
\hline & Highland & S08000022 & 95 & 87 & 79 & 73 & 74 & 68 \\
\hline & Lanarkshire & S08000023 & 207 & 217 & 213 & 236 & 227 & 219 \\
\hline & Lothian & S08000024 & 221 & 214 & 199 & 213 & 223 & 221 \\
\hline & Orkney & S08000025 & 6 & 8 & 6 & 6 & 8 & 6 \\
\hline & Shetland & S08000026 & 3 & 4 & 4 & 3 & 3 & 4 \\
\hline & Tayside & S08000027 & 161 & 170 & 176 & 167 & 161 & 158 \\
\hline & Western Isles & S08000028 & 8 & 11 & 8 & 6 & 5 & 8 \\
\hline \multirow[t]{5}{*}{ Northern Ireland } & Belfast & ZC010 & 141 & 144 & 141 & 138 & 137 & 136 \\
\hline & Northern & ZC020 & 174 & 170 & 183 & 184 & 181 & 181 \\
\hline & Southern & ZC030 & 113 & 123 & 127 & 100 & 99 & 100 \\
\hline & South Eastern & ZC040 & 105 & 93 & 95 & 99 & 91 & 83 \\
\hline & Western & ZC050 & 126 & 125 & 114 & 97 & 79 & 83 \\
\hline
\end{tabular}


Table F3.3. Number of prevalent patients on home-therapies by year and CCG/HB

Blank cells are values of 1 or 2 - these have been suppressed

\begin{tabular}{|c|c|c|c|c|c|c|c|c|}
\hline \multirow[b]{2}{*}{ UK Area } & \multirow[b]{2}{*}{ CCG/HB Name } & \multirow[b]{2}{*}{ Code } & \multicolumn{6}{|c|}{ Prevalent numbers on home-therapies } \\
\hline & & & 2009 & 2010 & 2011 & 2012 & 2013 & 2014 \\
\hline \multirow{6}{*}{$\begin{array}{l}\text { Cheshire, Warrington } \\
\text { and Wirral }\end{array}$} & NHS Eastern Cheshire & E38000056 & 26 & 18 & 21 & 21 & 19 & 19 \\
\hline & NHS South Cheshire & E38000151 & 16 & 12 & 12 & 17 & 18 & 17 \\
\hline & NHS Vale Royal & E38000189 & 13 & 13 & 13 & 10 & 8 & 6 \\
\hline & NHS Warrington & E38000194 & 14 & 15 & 15 & 14 & 13 & 16 \\
\hline & NHS West Cheshire & E38000196 & 16 & 20 & 19 & 20 & 21 & 18 \\
\hline & NHS Wirral & E38000208 & 17 & 14 & 21 & 14 & 18 & 10 \\
\hline \multirow{5}{*}{$\begin{array}{l}\text { Durham, Darlington } \\
\text { and Tees }\end{array}$} & NHS Darlington & E38000042 & 3 & 3 & 4 & & 3 & 3 \\
\hline & NHS Durham Dales, Easington and Sedgefield & E38000047 & 9 & 14 & 13 & 11 & 10 & 11 \\
\hline & NHS Hartlepool and Stockton-on-Tees & E38000075 & 7 & 7 & 8 & 8 & 8 & 10 \\
\hline & NHS North Durham & E38000116 & 8 & 14 & 13 & 17 & 8 & 12 \\
\hline & NHS South Tees & E38000162 & 4 & 4 & 3 & 5 & 6 & 6 \\
\hline \multirow[t]{12}{*}{ Greater Manchester } & NHS Bolton & E38000016 & 19 & 27 & 25 & 26 & 24 & 21 \\
\hline & NHS Bury & E38000024 & 23 & 18 & 20 & 21 & 19 & 17 \\
\hline & NHS Central Manchester & E38000032 & 21 & 19 & 17 & 14 & 15 & 17 \\
\hline & NHS Heywood, Middleton \& Rochdale & E38000080 & 16 & 16 & 23 & 20 & 18 & 25 \\
\hline & NHS North Manchester & E38000123 & 17 & 15 & 9 & 10 & 12 & 12 \\
\hline & NHS Oldham & E38000135 & 16 & 18 & 21 & 18 & 13 & 13 \\
\hline & NHS Salford & E38000143 & 16 & 12 & 13 & 10 & 16 & 13 \\
\hline & NHS South Manchester & E38000158 & 14 & 13 & 12 & 14 & 11 & 11 \\
\hline & NHS Stockport & E38000174 & 37 & 34 & 36 & 34 & 30 & 23 \\
\hline & NHS Tameside and Glossop & E38000182 & 22 & 24 & 28 & 23 & 24 & 20 \\
\hline & NHS Trafford & E38000187 & 16 & 24 & 23 & 22 & 19 & 13 \\
\hline & NHS Wigan Borough & E38000205 & 23 & 28 & 26 & 28 & 25 & 23 \\
\hline \multirow[t]{8}{*}{ Lancashire } & NHS Blackburn with Darwen & E38000014 & 14 & 12 & 15 & 11 & 3 & \\
\hline & NHS Blackpool & E38000015 & 7 & 7 & 7 & 7 & 7 & 8 \\
\hline & NHS Chorley and South Ribble & $\mathrm{E} 38000034$ & 19 & 12 & 13 & 13 & 10 & 11 \\
\hline & NHS East Lancashire & E38000050 & 24 & 25 & 31 & 29 & 25 & 26 \\
\hline & NHS Fylde \& Wyre & E38000060 & 11 & 11 & 10 & 9 & 12 & 12 \\
\hline & NHS Greater Preston & E38000065 & 8 & 6 & 5 & 12 & 10 & 16 \\
\hline & NHS Lancashire North & E38000093 & 8 & 8 & 10 & 17 & 17 & 11 \\
\hline & NHS West Lancashire & E38000200 & 9 & 6 & 6 & 4 & 8 & 8 \\
\hline \multirow[t]{6}{*}{ Merseyside } & NHS Halton & E38000068 & 8 & 8 & 12 & 14 & 13 & 11 \\
\hline & NHS Knowsley & E38000091 & 11 & 11 & 7 & 13 & 12 & 18 \\
\hline & NHS Liverpool & E38000101 & 34 & 29 & 30 & 32 & 34 & 37 \\
\hline & NHS South Sefton & E38000161 & 10 & 15 & 11 & 17 & 18 & 20 \\
\hline & NHS Southport and Formby & $\mathrm{E} 38000170$ & 7 & 8 & 10 & 8 & 11 & 11 \\
\hline & NHS St Helens & E38000172 & 16 & 15 & 14 & 22 & 21 & 18 \\
\hline \multirow{8}{*}{$\begin{array}{l}\text { Cumbria, Northumberland, } \\
\text { Tyne and Wear }\end{array}$} & NHS Cumbria & E38000041 & 30 & 29 & 37 & 37 & 36 & 40 \\
\hline & NHS Gateshead & E38000061 & 10 & 11 & 14 & 15 & 13 & 14 \\
\hline & NHS Newcastle North and East & E38000111 & 8 & 6 & 5 & 6 & 3 & 4 \\
\hline & NHS Newcastle West & E38000112 & 5 & 4 & 4 & 6 & 9 & 9 \\
\hline & NHS North Tyneside & E38000127 & 15 & 14 & 11 & 13 & 11 & 11 \\
\hline & NHS Northumberland & E38000130 & 19 & 23 & 15 & 21 & 21 & 24 \\
\hline & NHS South Tyneside & E38000163 & 11 & 12 & 9 & 8 & & 6 \\
\hline & NHS Sunderland & E38000176 & 16 & 16 & 8 & 6 & 7 & 6 \\
\hline
\end{tabular}


Table F3.3. Continued

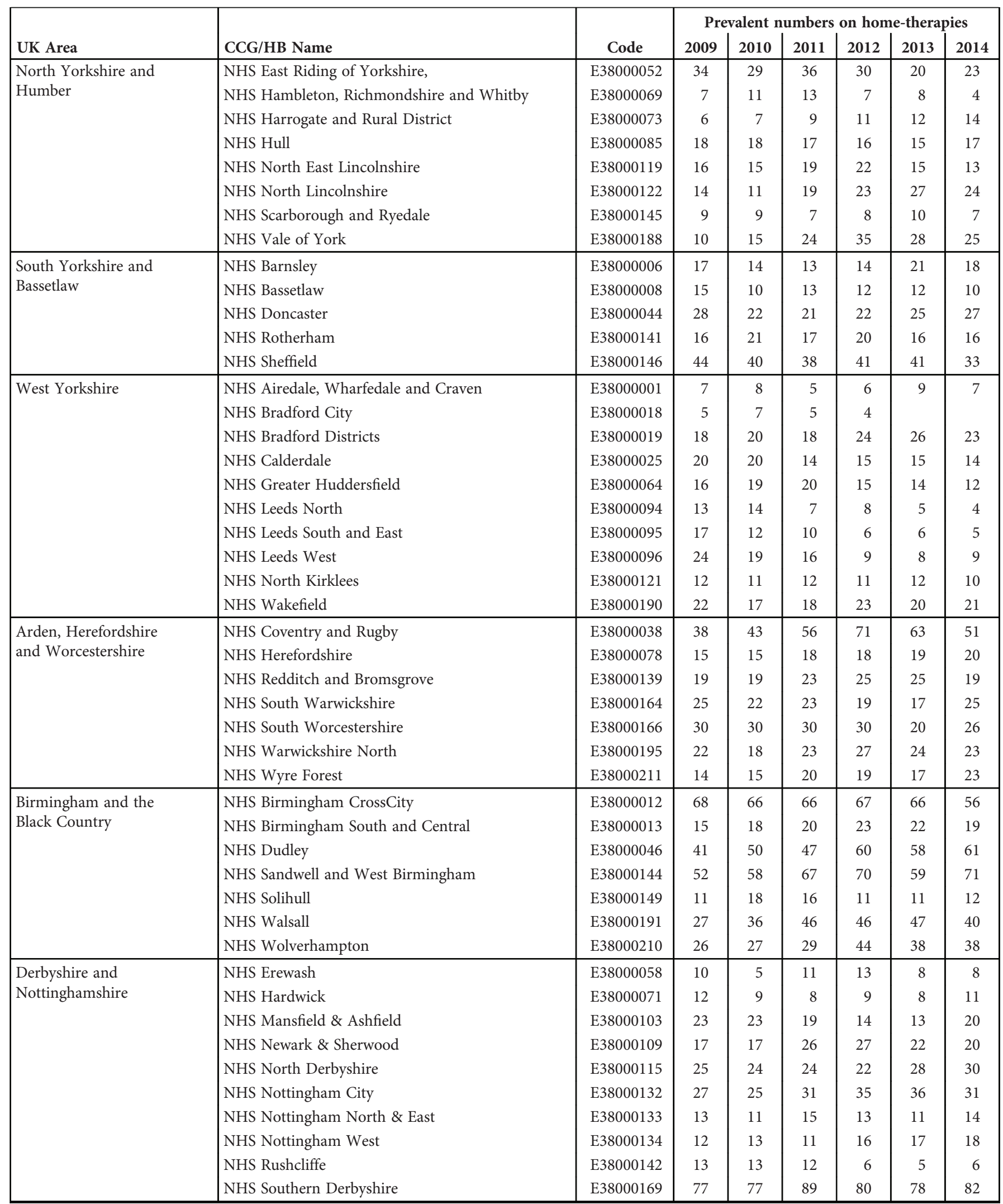


Table F3.3. Continued

\begin{tabular}{|c|c|c|c|c|c|c|c|c|}
\hline \multirow[b]{2}{*}{ UK Area } & \multirow[b]{2}{*}{ CCG/HB Name } & \multirow[b]{2}{*}{ Code } & \multicolumn{6}{|c|}{ Prevalent numbers on home-therapies } \\
\hline & & & 2009 & 2010 & 2011 & 2012 & 2013 & 2014 \\
\hline \multirow[t]{8}{*}{ East Anglia } & NHS Cambridgeshire and Peterborough & E38000026 & 34 & 36 & 45 & 41 & 42 & 42 \\
\hline & NHS Great Yarmouth \& Waveney & E38000063 & 10 & 14 & 18 & 15 & 13 & 13 \\
\hline & NHS Ipswich and East Suffolk & E38000086 & 38 & 29 & 24 & 23 & 24 & 28 \\
\hline & NHS North Norfolk & E38000124 & 19 & 20 & 16 & 21 & 15 & 14 \\
\hline & NHS Norwich & E38000131 & 14 & 12 & 11 & 10 & 12 & 16 \\
\hline & NHS South Norfolk & E38000159 & 21 & 15 & 28 & 26 & 24 & 19 \\
\hline & NHS West Norfolk & E38000203 & 12 & 13 & 14 & 8 & 6 & 8 \\
\hline & NHS West Suffolk & E38000204 & 13 & 12 & 10 & 17 & 14 & 13 \\
\hline \multirow[t]{7}{*}{ Essex } & NHS Basildon and Brentwood & E38000007 & 21 & 18 & 18 & 17 & 16 & 16 \\
\hline & NHS Castle Point, Rayleigh and Rochford & $\mathrm{E} 38000030$ & 9 & 11 & 11 & 10 & 10 & 13 \\
\hline & NHS Mid Essex & E38000106 & 24 & 25 & 21 & 21 & 20 & 20 \\
\hline & NHS North East Essex & E38000117 & 20 & 16 & 16 & 15 & 12 & 12 \\
\hline & NHS Southend & E38000168 & 10 & 10 & 11 & 9 & 12 & 10 \\
\hline & NHS Thurrock & E38000185 & 10 & 10 & 11 & 11 & 16 & 13 \\
\hline & NHS West Essex & E38000197 & 17 & 12 & 7 & 15 & 12 & 16 \\
\hline \multirow{7}{*}{$\begin{array}{l}\text { Hertfordshire and the } \\
\text { South Midlands }\end{array}$} & NHS Bedfordshire & E38000010 & 21 & 24 & 27 & 28 & 28 & 21 \\
\hline & NHS Corby & E38000037 & 3 & & 4 & 5 & 6 & 7 \\
\hline & NHS East and North Hertfordshire & E38000049 & 17 & 21 & 22 & 21 & 29 & 30 \\
\hline & NHS Herts Valleys & E38000079 & 14 & 13 & 10 & 18 & 24 & 24 \\
\hline & NHS Luton & E38000102 & 5 & 8 & 9 & 10 & 20 & 15 \\
\hline & NHS Milton Keynes & E38000107 & 18 & 19 & 17 & 16 & 19 & 15 \\
\hline & NHS Nene & E38000108 & 53 & 43 & 50 & 63 & 60 & 43 \\
\hline \multirow{7}{*}{$\begin{array}{l}\text { Leicestershire and } \\
\text { Lincolnshire }\end{array}$} & NHS East Leicestershire and Rutland & E38000051 & 21 & 23 & 26 & 25 & 28 & 22 \\
\hline & NHS Leicester City & E38000097 & 29 & 31 & 25 & 31 & 23 & 27 \\
\hline & NHS Lincolnshire East & E38000099 & 24 & 23 & 21 & 26 & 33 & 25 \\
\hline & NHS Lincolnshire West & $\mathrm{E} 38000100$ & 18 & 22 & 22 & 20 & 18 & 24 \\
\hline & NHS South Lincolnshire & E38000157 & 7 & 10 & 12 & 12 & 14 & 13 \\
\hline & NHS South West Lincolnshire & E38000165 & 10 & 14 & 15 & 14 & 13 & 11 \\
\hline & NHS West Leicestershire & E38000201 & 31 & 28 & 29 & 26 & 30 & 29 \\
\hline \multirow{8}{*}{$\begin{array}{l}\text { Shropshire and } \\
\text { Staffordshire }\end{array}$} & NHS Cannock Chase & E38000028 & 12 & 13 & 18 & 19 & 22 & 27 \\
\hline & NHS East Staffordshire & E38000053 & 12 & 18 & 21 & 18 & 16 & 17 \\
\hline & NHS North Staffordshire & E38000126 & 23 & 26 & 33 & 34 & 32 & 35 \\
\hline & NHS Shropshire & E38000147 & 23 & 20 & 35 & 35 & 31 & 34 \\
\hline & NHS South East Staffs and Seisdon and Peninsular & E38000153 & 17 & 19 & 27 & 27 & 22 & 18 \\
\hline & NHS Stafford and Surrounds & E38000173 & 11 & 21 & 17 & 23 & 23 & 23 \\
\hline & NHS Stoke on Trent & E38000175 & 21 & 24 & 27 & 24 & 27 & 28 \\
\hline & NHS Telford \& Wrekin & E38000183 & 7 & 8 & 11 & 21 & 20 & 16 \\
\hline \multirow[t]{11}{*}{ London } & NHS Barking \& Dagenham & E38000004 & 23 & 26 & 20 & 29 & 25 & 31 \\
\hline & NHS Barnet & E38000005 & 26 & 32 & 37 & 36 & 36 & 39 \\
\hline & NHS Camden & E38000027 & 7 & 8 & 9 & 11 & 11 & 13 \\
\hline & NHS City and Hackney & E38000035 & 7 & 13 & 15 & 19 & 26 & 21 \\
\hline & NHS Enfield & E38000057 & 19 & 18 & 22 & 29 & 30 & 36 \\
\hline & NHS Haringey & E38000072 & 6 & 5 & 14 & 23 & 27 & 26 \\
\hline & NHS Havering & E38000077 & 21 & 17 & 17 & 29 & 25 & 25 \\
\hline & NHS Islington & E38000088 & 6 & 8 & 11 & 17 & 19 & 21 \\
\hline & NHS Newham & E38000113 & 30 & 37 & 43 & 45 & 34 & 40 \\
\hline & NHS Redbridge & E38000138 & 47 & 41 & 29 & 32 & 37 & 46 \\
\hline & NHS Tower Hamlets & E38000186 & 18 & 22 & 20 & 24 & 26 & 27 \\
\hline
\end{tabular}


Table F3.3. Continued

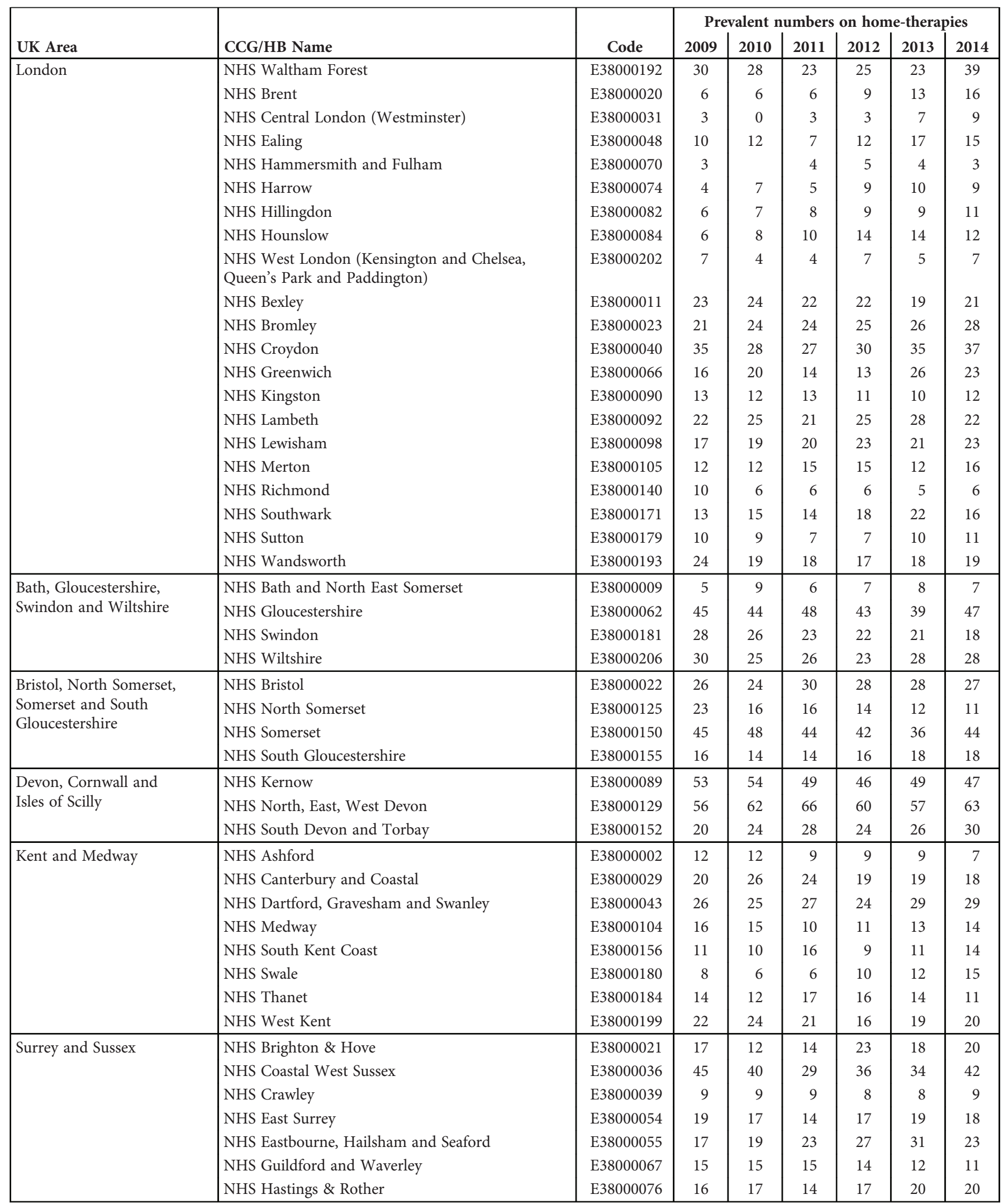


Table F3.3. Continued

\begin{tabular}{|c|c|c|c|c|c|c|c|c|}
\hline \multirow[b]{2}{*}{ UK Area } & \multirow[b]{2}{*}{ CCG/HB Name } & \multirow[b]{2}{*}{ Code } & \multicolumn{6}{|c|}{ Prevalent numbers on home-therapies } \\
\hline & & & 2009 & 2010 & 2011 & 2012 & 2013 & 2014 \\
\hline \multirow[t]{5}{*}{ Surrey and Sussex } & NHS High Weald Lewes Havens & E38000081 & 17 & 12 & 13 & 13 & 10 & 9 \\
\hline & NHS Horsham and Mid Sussex & E38000083 & 17 & 18 & 23 & 16 & 15 & 17 \\
\hline & NHS North West Surrey & E38000128 & 13 & 12 & 20 & 23 & 26 & 31 \\
\hline & NHS Surrey Downs & E38000177 & 23 & 24 & 29 & 31 & 26 & 23 \\
\hline & NHS Surrey Heath & E38000178 & 6 & 5 & 5 & 7 & 7 & 10 \\
\hline \multirow[t]{10}{*}{ Thames Valley } & NHS Aylesbury Vale & E38000003 & 7 & 9 & 12 & 5 & 8 & 4 \\
\hline & NHS Bracknell and Ascot & E38000017 & 8 & 8 & 5 & 3 & 8 & 10 \\
\hline & NHS Chiltern & E38000033 & 31 & 28 & 15 & 11 & 16 & 16 \\
\hline & NHS Newbury and District & E38000110 & 9 & 7 & 7 & 11 & 8 & 9 \\
\hline & NHS North \& West Reading & E38000114 & 10 & 9 & 11 & 9 & 7 & 8 \\
\hline & NHS Oxfordshire & E38000136 & 40 & 38 & 38 & 39 & 48 & 40 \\
\hline & NHS Slough & E38000148 & 21 & 19 & 20 & 23 & 19 & 13 \\
\hline & NHS South Reading & E38000160 & 13 & 15 & 14 & 13 & 16 & 17 \\
\hline & NHS Windsor, Ascot and Maidenhead & E38000207 & 10 & 8 & 14 & 7 & 7 & 14 \\
\hline & NHS Wokingham & E38000209 & 5 & 6 & 10 & 10 & 13 & 9 \\
\hline \multirow[t]{9}{*}{ Wessex } & NHS Dorset & E38000045 & 55 & 51 & 43 & 45 & 51 & 54 \\
\hline & NHS Fareham and Gosport & E38000059 & 15 & 20 & 19 & 23 & 17 & 20 \\
\hline & NHS Isle of Wight & E38000087 & 5 & 4 & 4 & & 6 & 9 \\
\hline & NHS North East Hampshire and Farnham & E38000118 & 12 & 12 & 9 & 9 & 11 & 16 \\
\hline & NHS North Hampshire & E38000120 & 10 & 9 & 6 & 7 & 16 & 17 \\
\hline & NHS Portsmouth & E38000137 & 4 & 3 & 6 & 8 & 12 & 9 \\
\hline & NHS South Eastern Hampshire & E38000154 & 13 & 10 & 13 & 11 & 13 & 7 \\
\hline & NHS Southampton & E38000167 & 8 & 12 & 10 & 8 & 7 & 11 \\
\hline & NHS West Hampshire & E38000198 & 37 & 40 & 33 & 27 & 30 & 31 \\
\hline \multirow[t]{7}{*}{ Wales } & Betsi Cadwaladr University & W11000023 & 73 & 72 & 68 & 74 & 71 & 76 \\
\hline & Powys Teaching & W11000024 & 10 & 9 & 15 & 14 & 12 & 14 \\
\hline & Hywel Dda & W11000025 & 30 & 27 & 34 & 35 & 34 & 42 \\
\hline & Abertawe Bro Morgannwg University & W11000026 & 56 & 56 & 55 & 62 & 49 & 54 \\
\hline & Cwm Taf & W11000027 & 38 & 34 & 37 & 27 & 25 & 26 \\
\hline & Aneurin Bevan & W11000028 & 53 & 56 & 51 & 50 & 48 & 49 \\
\hline & Cardiff and Vale University & W11000029 & 30 & 26 & 30 & 19 & 26 & 30 \\
\hline \multirow[t]{14}{*}{ Scotland } & Ayrshire and Arran & S08000015 & 44 & 49 & 54 & 50 & 50 & 47 \\
\hline & Borders & S08000016 & 7 & 7 & 5 & 5 & 5 & 4 \\
\hline & Dumfries and Galloway & S08000017 & 14 & 8 & 14 & 17 & 18 & 18 \\
\hline & Fife & S08000018 & 30 & 30 & 31 & 21 & 23 & 17 \\
\hline & Forth Valley & S08000019 & 18 & 17 & 12 & 14 & 11 & 13 \\
\hline & Grampian & S08000020 & 32 & 33 & 26 & 28 & 32 & 33 \\
\hline & Greater Glasgow and Clyde & S08000021 & 47 & 49 & 52 & 52 & 42 & 39 \\
\hline & Highland & S08000022 & 30 & 29 & 26 & 30 & 22 & 22 \\
\hline & Lanarkshire & S08000023 & 23 & 18 & 18 & 18 & 21 & 15 \\
\hline & Lothian & S08000024 & 56 & 48 & 39 & 41 & 28 & 23 \\
\hline & Orkney & S08000025 & 3 & & & & & \\
\hline & Shetland & S08000026 & & & & & & \\
\hline & Tayside & S08000027 & 20 & 18 & 15 & 18 & 17 & 22 \\
\hline & Western Isles & S08000028 & & & & & & 3 \\
\hline \multirow[t]{5}{*}{ Northern Ireland } & Belfast & ZC010 & 15 & 9 & 13 & 20 & 19 & 12 \\
\hline & Northern & ZC020 & 27 & 24 & 27 & 29 & 26 & 22 \\
\hline & Southern & ZC030 & 26 & 23 & 21 & 27 & 28 & 22 \\
\hline & South Eastern & ZC040 & 17 & 20 & 21 & 18 & 17 & 12 \\
\hline & Western & ZC050 & 13 & 13 & 22 & 21 & 19 & 15 \\
\hline
\end{tabular}


Table F3.4 Number of prevalent patients on transplant by year and CCG/HB

\begin{tabular}{|c|c|c|c|c|c|c|c|c|}
\hline \multirow[b]{2}{*}{ UK area } & \multirow[b]{2}{*}{ CCG/HB name } & \multirow[b]{2}{*}{ Code } & \multicolumn{6}{|c|}{ Prevalent numbers on transplant } \\
\hline & & & 2009 & 2010 & 2011 & 2012 & 2013 & 2014 \\
\hline \multirow{6}{*}{$\begin{array}{l}\text { Cheshire, Warrington } \\
\text { and Wirral }\end{array}$} & NHS Eastern Cheshire & E38000056 & 59 & 70 & 76 & 81 & 87 & 91 \\
\hline & NHS South Cheshire & E38000151 & 60 & 69 & 69 & 71 & 79 & 89 \\
\hline & NHS Vale Royal & E38000189 & 28 & 28 & 29 & 32 & 37 & 38 \\
\hline & NHS Warrington & E38000194 & 81 & 74 & 78 & 83 & 95 & 99 \\
\hline & NHS West Cheshire & E38000196 & 82 & 88 & 93 & 98 & 106 & 113 \\
\hline & NHS Wirral & E38000208 & 108 & 110 & 112 & 111 & 116 & 118 \\
\hline \multirow{5}{*}{$\begin{array}{l}\text { Durham, Darlington } \\
\text { and Tees }\end{array}$} & NHS Darlington & E38000042 & 33 & 35 & 41 & 42 & 47 & 52 \\
\hline & NHS Durham Dales, Easington and Sedgefield & E38000047 & 110 & 112 & 123 & 126 & 138 & 150 \\
\hline & NHS Hartlepool and Stockton-on-Tees & E38000075 & 117 & 123 & 118 & 125 & 134 & 142 \\
\hline & NHS North Durham & E38000116 & 91 & 97 & 96 & 100 & 103 & 105 \\
\hline & NHS South Tees & E38000162 & 142 & 144 & 155 & 158 & 158 & 163 \\
\hline \multirow[t]{12}{*}{ Greater Manchester } & NHS Bolton & E38000016 & 119 & 127 & 140 & 149 & 153 & 161 \\
\hline & NHS Bury & E38000024 & 72 & 73 & 76 & 82 & 83 & 92 \\
\hline & NHS Central Manchester & E38000032 & 52 & 60 & 64 & 67 & 78 & 85 \\
\hline & NHS Heywood, Middleton \& Rochdale & E38000080 & 82 & 85 & 93 & 98 & 104 & 99 \\
\hline & NHS North Manchester & E38000123 & 39 & 46 & 52 & 58 & 64 & 69 \\
\hline & NHS Oldham & E38000135 & 81 & 87 & 91 & 93 & 106 & 105 \\
\hline & NHS Salford & E38000143 & 72 & 81 & 86 & 98 & 99 & 106 \\
\hline & NHS South Manchester & E38000158 & 32 & 38 & 45 & 51 & 55 & 61 \\
\hline & NHS Stockport & E38000174 & 108 & 115 & 118 & 123 & 131 & 133 \\
\hline & NHS Tameside and Glossop & E38000182 & 98 & 104 & 114 & 116 & 121 & 130 \\
\hline & NHS Trafford & E38000187 & 66 & 75 & 80 & 87 & 93 & 105 \\
\hline & NHS Wigan Borough & E38000205 & 112 & 126 & 147 & 157 & 174 & 178 \\
\hline \multirow[t]{8}{*}{ Lancashire } & NHS Blackburn with Darwen & E38000014 & 48 & 49 & 56 & 60 & 67 & 73 \\
\hline & NHS Blackpool & E38000015 & 51 & 49 & 49 & 57 & 68 & 74 \\
\hline & NHS Chorley and South Ribble & E38000034 & 52 & 60 & 69 & 69 & 76 & 80 \\
\hline & NHS East Lancashire & E38000050 & 152 & 152 & 164 & 166 & 177 & 183 \\
\hline & NHS Fylde \& Wyre & $\mathrm{E} 38000060$ & 56 & 55 & 57 & 64 & 69 & 70 \\
\hline & NHS Greater Preston & E38000065 & 63 & 64 & 66 & 75 & 79 & 85 \\
\hline & NHS Lancashire North & E38000093 & 51 & 52 & 53 & 53 & 55 & 58 \\
\hline & NHS West Lancashire & E38000200 & 34 & 38 & 40 & 43 & 43 & 44 \\
\hline \multirow[t]{6}{*}{ Merseyside } & NHS Halton & E38000068 & 44 & 49 & 52 & 57 & 58 & 63 \\
\hline & NHS Knowsley & E38000091 & 54 & 56 & 55 & 58 & 61 & 62 \\
\hline & NHS Liverpool & E38000101 & 152 & 163 & 176 & 184 & 196 & 209 \\
\hline & NHS South Sefton & E38000161 & 54 & 57 & 60 & 67 & 72 & 73 \\
\hline & NHS Southport and Formby & E38000170 & 29 & 35 & 36 & 33 & 40 & 41 \\
\hline & NHS St Helens & E38000172 & 54 & 59 & 63 & 64 & 72 & 82 \\
\hline \multirow{8}{*}{$\begin{array}{l}\text { Cumbria, Northumberland, } \\
\text { Tyne and Wear }\end{array}$} & NHS Cumbria & E38000041 & 185 & 197 & 201 & 213 & 227 & 238 \\
\hline & NHS Gateshead & E38000061 & 77 & 77 & 84 & 88 & 87 & 88 \\
\hline & NHS Newcastle North and East & E38000111 & 60 & 61 & 67 & 63 & 65 & 70 \\
\hline & NHS Newcastle West & E38000112 & 47 & 44 & 46 & 48 & 51 & 52 \\
\hline & NHS North Tyneside & E38000127 & 105 & 114 & 117 & 117 & 117 & 111 \\
\hline & NHS Northumberland & E38000130 & 129 & 121 & 135 & 138 & 150 & 156 \\
\hline & NHS South Tyneside & E38000163 & 70 & 70 & 75 & 76 & 83 & 77 \\
\hline & NHS Sunderland & E38000176 & 113 & 119 & 129 & 136 & 142 & 144 \\
\hline
\end{tabular}


Table F3.4 Continued

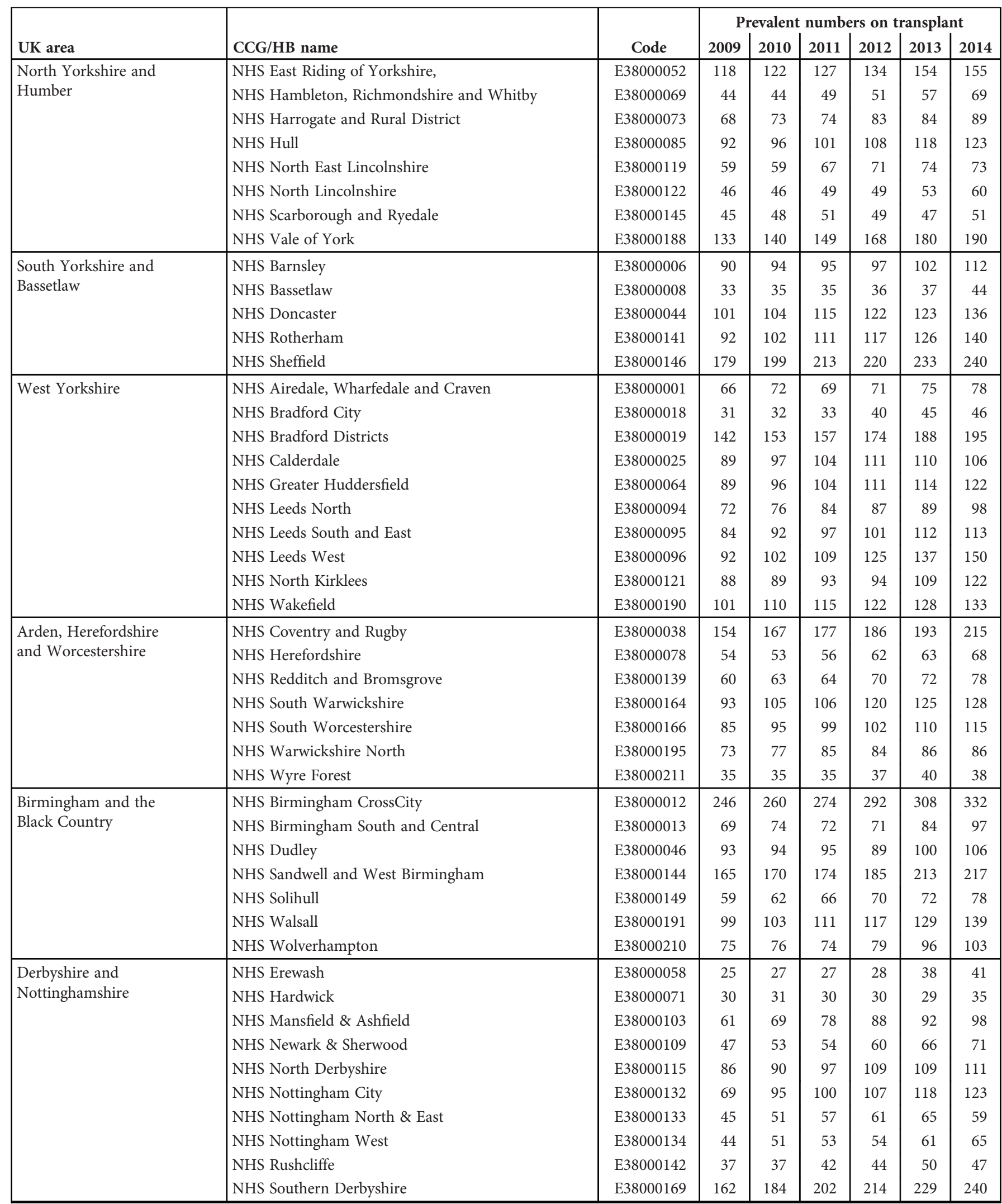


Table F3.4 Continued

\begin{tabular}{|c|c|c|c|c|c|c|c|c|}
\hline \multirow[b]{2}{*}{ UK area } & \multirow[b]{2}{*}{ CCG/HB name } & \multirow[b]{2}{*}{ Code } & \multicolumn{6}{|c|}{ Prevalent numbers on transplant } \\
\hline & & & 2009 & 2010 & 2011 & 2012 & 2013 & 2014 \\
\hline \multirow[t]{8}{*}{ East Anglia } & NHS Cambridgeshire and Peterborough & E38000026 & 294 & 317 & 339 & 349 & 370 & 391 \\
\hline & NHS Great Yarmouth \& Waveney & E38000063 & 64 & 64 & 67 & 72 & 92 & 102 \\
\hline & NHS Ipswich and East Suffolk & E38000086 & 121 & 133 & 146 & 147 & 170 & 176 \\
\hline & NHS North Norfolk & E38000124 & 63 & 62 & 68 & 63 & 84 & 81 \\
\hline & NHS Norwich & E38000131 & 52 & 54 & 59 & 58 & 76 & 80 \\
\hline & NHS South Norfolk & E38000159 & 84 & 91 & 86 & 91 & 113 & 116 \\
\hline & NHS West Norfolk & E38000203 & 57 & 57 & 58 & 65 & 68 & 74 \\
\hline & NHS West Suffolk & E38000204 & 76 & 81 & 83 & 91 & 92 & 93 \\
\hline \multirow[t]{7}{*}{ Essex } & NHS Basildon and Brentwood & E38000007 & 81 & 92 & 97 & 98 & 120 & 120 \\
\hline & NHS Castle Point, Rayleigh and Rochford & E38000030 & 65 & 62 & 62 & 64 & 72 & 85 \\
\hline & NHS Mid Essex & E38000106 & 141 & 148 & 163 & 159 & 180 & 183 \\
\hline & NHS North East Essex & E38000117 & 103 & 109 & 120 & 125 & 137 & 155 \\
\hline & NHS Southend & E38000168 & 53 & 60 & 63 & 71 & 81 & 87 \\
\hline & NHS Thurrock & E38000185 & 49 & 52 & 58 & 60 & 61 & 65 \\
\hline & NHS West Essex & E38000197 & 95 & 106 & 108 & 119 & 123 & 133 \\
\hline \multirow{7}{*}{$\begin{array}{l}\text { Hertfordshire and the } \\
\text { South Midlands }\end{array}$} & NHS Bedfordshire & E38000010 & 161 & 172 & 176 & 200 & 208 & 225 \\
\hline & NHS Corby & E38000037 & 20 & 21 & 23 & 21 & 21 & 22 \\
\hline & NHS East and North Hertfordshire & E38000049 & 178 & 195 & 203 & 220 & 234 & 250 \\
\hline & NHS Herts Valleys & E38000079 & 194 & 221 & 234 & 241 & 258 & 279 \\
\hline & NHS Luton & E38000102 & 71 & 79 & 90 & 98 & 109 & 124 \\
\hline & NHS Milton Keynes & E38000107 & 88 & 98 & 108 & 116 & 117 & 133 \\
\hline & NHS Nene & E38000108 & 228 & 246 & 256 & 252 & 269 & 297 \\
\hline \multirow{7}{*}{$\begin{array}{l}\text { Leicestershire and } \\
\text { Lincolnshire }\end{array}$} & NHS East Leicestershire and Rutland & E38000051 & 118 & 120 & 126 & 132 & 139 & 155 \\
\hline & NHS Leicester City & E38000097 & 168 & 168 & 179 & 187 & 206 & 226 \\
\hline & NHS Lincolnshire East & E38000099 & 77 & 84 & 85 & 90 & 97 & 102 \\
\hline & NHS Lincolnshire West & E38000100 & 75 & 75 & 79 & 82 & 91 & 97 \\
\hline & NHS South Lincolnshire & E38000157 & 34 & 40 & 40 & 44 & 44 & 52 \\
\hline & NHS South West Lincolnshire & E38000165 & 32 & 31 & 38 & 41 & 44 & 46 \\
\hline & NHS West Leicestershire & E38000201 & 145 & 158 & 168 & 174 & 182 & 189 \\
\hline \multirow{8}{*}{$\begin{array}{l}\text { Shropshire and } \\
\text { Staffordshire }\end{array}$} & NHS Cannock Chase & E38000028 & 46 & 45 & 44 & 44 & 48 & 49 \\
\hline & NHS East Staffordshire & E38000053 & 26 & 29 & 32 & 31 & 41 & 41 \\
\hline & NHS North Staffordshire & E38000126 & 75 & 76 & 82 & 88 & 95 & 95 \\
\hline & NHS Shropshire & E38000147 & 105 & 107 & 111 & 106 & 110 & 114 \\
\hline & NHS South East Staffs and Seisdon and Peninsular & E38000153 & 81 & 90 & 88 & 85 & 95 & 101 \\
\hline & NHS Stafford and Surrounds & E38000173 & 48 & 48 & 52 & 55 & 61 & 66 \\
\hline & NHS Stoke on Trent & E38000175 & 99 & 106 & 105 & 112 & 112 & 119 \\
\hline & NHS Telford \& Wrekin & E38000183 & 47 & 48 & 49 & 48 & 56 & 56 \\
\hline \multirow[t]{11}{*}{ London } & NHS Barking \& Dagenham & E38000004 & 59 & 64 & 76 & 75 & 88 & 94 \\
\hline & NHS Barnet & E38000005 & 159 & 171 & 190 & 214 & 224 & 227 \\
\hline & NHS Camden & E38000027 & 87 & 90 & 101 & 107 & 108 & 109 \\
\hline & NHS City and Hackney & E38000035 & 83 & 87 & 87 & 91 & 104 & 117 \\
\hline & NHS Enfield & E38000057 & 143 & 155 & 174 & 191 & 198 & 215 \\
\hline & NHS Haringey & $\mathrm{E} 38000072$ & 103 & 111 & 121 & 132 & 139 & 153 \\
\hline & NHS Havering & E38000077 & 72 & 75 & 79 & 82 & 95 & 93 \\
\hline & NHS Islington & E38000088 & 97 & 99 & 107 & 116 & 122 & 130 \\
\hline & NHS Newham & E38000113 & 84 & 97 & 101 & 117 & 137 & 158 \\
\hline & NHS Redbridge & E38000138 & 101 & 119 & 126 & 143 & 151 & 170 \\
\hline & NHS Tower Hamlets & E38000186 & 59 & 72 & 73 & 83 & 89 & 104 \\
\hline
\end{tabular}


Table F3.4 Continued

\begin{tabular}{|c|c|c|c|c|c|c|c|c|}
\hline \multirow[b]{2}{*}{ UK area } & \multirow[b]{2}{*}{ CCG/HB name } & \multirow[b]{2}{*}{ Code } & \multicolumn{6}{|c|}{ Prevalent numbers on transplant } \\
\hline & & & 2009 & 2010 & 2011 & 2012 & 2013 & 2014 \\
\hline \multirow[t]{16}{*}{ London } & NHS Waltham Forest & E38000192 & 99 & 108 & 115 & 115 & 125 & 144 \\
\hline & NHS Central London (Westminster) & E38000031 & 66 & 73 & 74 & 77 & 81 & 91 \\
\hline & NHS Ealing & E38000048 & 181 & 195 & 203 & 214 & 220 & 242 \\
\hline & NHS Hammersmith and Fulham & E38000070 & 69 & 77 & 75 & 78 & 84 & 88 \\
\hline & NHS Hillingdon & E38000082 & 134 & 144 & 159 & 168 & 170 & 188 \\
\hline & NHS Hounslow & E38000084 & 120 & 129 & 133 & 137 & 155 & 169 \\
\hline & $\begin{array}{l}\text { NHS West London (Kensington and Chelsea, } \\
\text { Queen's Park and Paddington) }\end{array}$ & E38000202 & 88 & 103 & 102 & 101 & 106 & 114 \\
\hline & NHS Bexley & E38000011 & 106 & 118 & 121 & 124 & 134 & 137 \\
\hline & NHS Bromley & E38000023 & 138 & 151 & 151 & 160 & 167 & 174 \\
\hline & NHS Lambeth & E38000092 & 96 & 97 & 110 & 124 & 139 & 152 \\
\hline & NHS Lewisham & E38000098 & 108 & 106 & 109 & 112 & 134 & 146 \\
\hline & NHS Merton & E38000105 & 81 & 82 & 88 & 96 & 109 & 115 \\
\hline & NHS Richmond & E38000140 & 56 & 59 & 64 & 69 & 75 & 80 \\
\hline & NHS Southwark & E38000171 & 131 & 140 & 149 & 163 & 177 & 190 \\
\hline & NHS Sutton & E38000179 & 78 & 85 & 88 & 95 & 97 & 98 \\
\hline & NHS Wandsworth & E38000193 & 95 & 102 & 113 & 120 & 129 & 141 \\
\hline \multirow{3}{*}{$\begin{array}{l}\text { Bath, Gloucestershire, } \\
\text { Swindon and Wiltshire }\end{array}$} & NHS Bath and North East Somerset & E38000009 & 53 & 51 & 51 & 52 & 65 & 72 \\
\hline & NHS Gloucestershire & E38000062 & 208 & 213 & 232 & 229 & 255 & 257 \\
\hline & NHS Swindon & E38000181 & 75 & 90 & 96 & 98 & 106 & 114 \\
\hline \multirow{2}{*}{$\begin{array}{l}\text { Devon, Cornwall } \\
\text { and Isles of Scilly }\end{array}$} & NHS North, East, West Devon & E38000129 & 366 & 371 & 377 & 398 & 429 & 440 \\
\hline & NHS South Devon and Torbay & E38000152 & 122 & 129 & 134 & 136 & 152 & 164 \\
\hline \multirow[t]{8}{*}{ Kent and Medway } & NHS Ashford & E38000002 & 54 & 56 & 59 & 65 & 65 & 70 \\
\hline & NHS Canterbury and Coastal & E38000029 & 76 & 81 & 86 & 100 & 102 & 112 \\
\hline & NHS Dartford, Gravesham and Swanley & E38000043 & 119 & 120 & 117 & 122 & 129 & 138 \\
\hline & NHS Medway & E38000104 & 103 & 110 & 111 & 117 & 127 & 130 \\
\hline & NHS South Kent Coast & E38000156 & 63 & 70 & 75 & 79 & 85 & 95 \\
\hline & NHS Swale & E38000180 & 45 & 46 & 57 & 60 & 67 & 68 \\
\hline & NHS Thanet & E38000184 & 50 & 56 & 63 & 74 & 81 & 86 \\
\hline & NHS West Kent & E38000199 & 161 & 162 & 169 & 180 & 191 & 201 \\
\hline \multirow[t]{7}{*}{ Surrey and Sussex } & NHS Brighton \& Hove & E38000021 & 88 & 97 & 99 & 101 & 103 & 108 \\
\hline & NHS Coastal West Sussex & E38000036 & 184 & 189 & 203 & 202 & 219 & 230 \\
\hline & NHS Crawley & E38000039 & 28 & 28 & 31 & 32 & 32 & 35 \\
\hline & NHS East Surrey & E38000054 & 57 & 58 & 60 & 61 & 67 & 65 \\
\hline & NHS Eastbourne, Hailsham and Seaford & E38000055 & 55 & 58 & 60 & 62 & 66 & 68 \\
\hline & NHS Guildford and Waverley & E38000067 & 60 & 59 & 56 & 64 & 67 & 70 \\
\hline & NHS Hastings \& Rother & E38000076 & 55 & 59 & 64 & 63 & 67 & 72 \\
\hline
\end{tabular}


Table F3.4 Continued

\begin{tabular}{|c|c|c|c|c|c|c|c|c|}
\hline \multirow[b]{2}{*}{ UK area } & \multirow[b]{2}{*}{ CCG/HB name } & \multirow[b]{2}{*}{ Code } & \multicolumn{6}{|c|}{ Prevalent numbers on transplant } \\
\hline & & & 2009 & 2010 & 2011 & 2012 & 2013 & 2014 \\
\hline \multirow[t]{5}{*}{ Surrey and Sussex } & NHS High Weald Lewes Havens & E38000081 & 54 & 56 & 57 & 67 & 68 & 72 \\
\hline & NHS Horsham and Mid Sussex & E38000083 & 69 & 73 & 73 & 74 & 80 & 92 \\
\hline & NHS North West Surrey & E38000128 & 140 & 143 & 146 & 154 & 162 & 168 \\
\hline & NHS Surrey Downs & E38000177 & 106 & 112 & 113 & 114 & 123 & 129 \\
\hline & NHS Surrey Heath & E38000178 & 43 & 45 & 48 & 51 & 48 & 44 \\
\hline \multirow[t]{10}{*}{ Thames Valley } & NHS Aylesbury Vale & E38000003 & 96 & 98 & 104 & 108 & 112 & 115 \\
\hline & NHS Bracknell and Ascot & E38000017 & 51 & 56 & 61 & 64 & 67 & 67 \\
\hline & NHS Chiltern & E38000033 & 127 & 136 & 135 & 150 & 159 & 159 \\
\hline & NHS Newbury and District & E38000110 & 57 & 53 & 60 & 60 & 61 & 60 \\
\hline & NHS North \& West Reading & E38000114 & 35 & 41 & 41 & 44 & 50 & 49 \\
\hline & NHS Oxfordshire & E38000136 & 258 & 276 & 285 & 306 & 315 & 339 \\
\hline & NHS Slough & E38000148 & 79 & 86 & 87 & 91 & 109 & 116 \\
\hline & NHS South Reading & E38000160 & 55 & 54 & 55 & 54 & 58 & 64 \\
\hline & NHS Windsor, Ascot and Maidenhead & E38000207 & 48 & 57 & 61 & 71 & 78 & 83 \\
\hline & NHS Wokingham & E38000209 & 62 & 64 & 66 & 70 & 71 & 76 \\
\hline \multirow[t]{9}{*}{ Wessex } & NHS Dorset & E38000045 & 297 & 305 & 311 & 309 & 316 & 333 \\
\hline & NHS Fareham and Gosport & E38000059 & 78 & 78 & 81 & 80 & 92 & 96 \\
\hline & NHS Isle of Wight & E38000087 & 47 & 49 & 50 & 52 & 49 & 49 \\
\hline & NHS North East Hampshire and Farnham & E38000118 & 68 & 76 & 76 & 80 & 86 & 94 \\
\hline & NHS North Hampshire & E38000120 & 69 & 72 & 78 & 81 & 84 & 88 \\
\hline & NHS Portsmouth & E38000137 & 67 & 77 & 77 & 80 & 85 & 85 \\
\hline & NHS South Eastern Hampshire & E38000154 & 81 & 87 & 85 & 91 & 94 & 107 \\
\hline & NHS Southampton & E38000167 & 80 & 79 & 90 & 98 & 108 & 117 \\
\hline & NHS West Hampshire & E38000198 & 206 & 218 & 225 & 231 & 240 & 244 \\
\hline \multirow[t]{7}{*}{ Wales } & Betsi Cadwaladr University & W11000023 & 240 & 250 & 250 & 246 & 236 & 248 \\
\hline & Powys Teaching & W11000024 & 50 & 55 & 54 & 47 & 50 & 51 \\
\hline & Hywel Dda & W11000025 & 155 & 154 & 165 & 163 & 187 & 189 \\
\hline & Abertawe Bro Morgannwg University & W11000026 & 234 & 255 & 284 & 299 & 313 & 319 \\
\hline & Cwm Taf & W11000027 & 168 & 186 & 196 & 203 & 219 & 216 \\
\hline & Aneurin Bevan & W11000028 & 270 & 289 & 302 & 337 & 346 & 350 \\
\hline & Cardiff and Vale University & W11000029 & 196 & 211 & 223 & 238 & 244 & 242 \\
\hline \multirow[t]{14}{*}{ Scotland } & Ayrshire and Arran & S08000015 & 149 & 147 & 144 & 154 & 162 & 173 \\
\hline & Borders & S08000016 & 46 & 53 & 53 & 60 & 62 & 63 \\
\hline & Dumfries and Galloway & S08000017 & 55 & 55 & 60 & 59 & 59 & 66 \\
\hline & Fife & S08000018 & 116 & 122 & 132 & 138 & 150 & 154 \\
\hline & Forth Valley & S08000019 & 89 & 95 & 103 & 111 & 119 & 133 \\
\hline & Grampian & S08000020 & 210 & 216 & 224 & 235 & 254 & 257 \\
\hline & Greater Glasgow and Clyde & S08000021 & 473 & 482 & 500 & 552 & 594 & 624 \\
\hline & Highland & S08000022 & 149 & 155 & 154 & 155 & 162 & 170 \\
\hline & Lanarkshire & S08000023 & 253 & 266 & 279 & 301 & 314 & 344 \\
\hline & Lothian & S08000024 & 280 & 295 & 309 & 318 & 327 & 348 \\
\hline & Orkney & S08000025 & 9 & 8 & 8 & 8 & 8 & 6 \\
\hline & Shetland & S08000026 & 6 & 6 & 5 & 6 & 6 & 6 \\
\hline & Tayside & S08000027 & 168 & 167 & 172 & 175 & 184 & 189 \\
\hline & Western Isles & S08000028 & 7 & 7 & 8 & 8 & 8 & 8 \\
\hline \multirow[t]{5}{*}{ Northern Ireland } & Belfast & $\mathrm{ZC} 010$ & 122 & 134 & 138 & 150 & 160 & 178 \\
\hline & Northern & $\mathrm{ZC} 020$ & 159 & 167 & 174 & 179 & 193 & 213 \\
\hline & Southern & ZC030 & 106 & 112 & 126 & 142 & 153 & 171 \\
\hline & South Eastern & $\mathrm{ZC} 040$ & 125 & 126 & 136 & 138 & 147 & 163 \\
\hline & Western & $\mathrm{ZC} 050$ & 97 & 103 & 105 & 107 & 130 & 155 \\
\hline
\end{tabular}


